Theorie der Involutionssysteme partieller Differentialgleichungen erster Ordnung in beliebig vielen abhängigen und unabhängigen Veränderlichen.

(Erste Abhandlung.)

Von

E. v. Weber in München.

Jedes beliebige System partieller Differentialgleichungen kann durch Einführung geeigneter abbängiger Variabeln in ein System von Gleichungen erster Ordnung verwandelt werden. Unter den letztgenannten Systemen beanspruchen die von $\mathrm{Lie}^{*}$ ) so bezeichneten "Involutionssysteme" das hauptsächlichste Interesse. Wir betrachten im Folgenden nur solche Systeme I. 0 . in den Independenten $x_{1} \ldots x_{m}$ und den unbekannten Functionen $z^{1} \ldots z^{n}$, die sich in gewisser Weise nach den Ableitungen der $z^{i}$ auflösen lassen $*$ ); die Involutionseigensehaft kann dann kurz dahin charakterisirt werden, dass ein solches System die grösstmögliche, mit seiner form verträgliche Mannigfaltigkeit von Integralen $z^{1} \cdot z^{n}$ besitzt. Die vorliegende erste $\mathrm{Ab}$ handlung beschäftigt sich mit folgenden Gegenständen:

Das erste Capitel behandelt die algebraischen Definitionsgleichnngen eines Involutionssystems, und die Frage nach der Existenz eines allgemeinen Integrals, wobei wir uns im Wesentlichen auf die grundlegenden Ontersuchungen des H. Bourlet*) stützen.

Das zweite Capitel ist der Betrachtung einer für die ganze Theorie fundamentalen Matrix gewidmet. Die Eigenschaften dieser Matrix führen uns in Cap. III zu einer Theorie der charakteristischen Mannigfaltigkeiten, insbesondere zur Definition einer besonderen Categorie von Involutionssystemen, der sog. "Normalsysteme". Für diese letzteren skizziren wir in Cap. IV eine Integrationstheorie, indem wir zeigen,

*) Vgl. Leipz. Ber. 47, p. 53-128 (1895).

*) Einen Specialfall hiervon untersacht Herr König, Math. Ann. 23, p. 520, den allgemeinen Fall für lineare Systeme Herr Bourlet, Ann. de l'Ec. Norm. (3) VIII (1891) Supplém. p. 43 ff. 
wie man unter der Annahme der Integrabilität gewisser totaler Differentialgleichungen eine Reduction der Anzahl der Independenten herbeiführen $k a n n^{*}$ ).

Die weitere Durchführung dieses Ansatzes, die Besprechung der mannigfachen Analogien, die unsere Theorie zu derjenigen der partiellen Differentialgleichungen I. O. mit einer Unbekannten darbietet, endich die Untersuchnng der linearen Involutionssysteme soll einer zweiten Abhandlung vorbehalten bleiben.

\section{Capitel I.}

Die Definitionsgleichungen and das allgemeine Integral des Involutionssystems.

1. Wir bezeichnèn mit $z^{1}, z^{2}, \ldots, z^{n}$ Functionen der $m$ unabhängigen Veränderlichen $x_{1}, x_{2}, \ldots, x_{m}$, setzen:

$$
p_{i}^{k} \equiv \frac{\partial z^{k}}{\partial x_{i}}
$$

und betrachten das folgende System partieller Differentialgleichungen erster Ordnung:

(A) $f_{1}\left(x_{1} \ldots x_{m}, z^{1} \ldots z^{n}, p_{1}^{1} \ldots p_{m}^{n}\right)=0, \quad f_{2}=0 \ldots f_{N}=0$.

Die Anzahl $N$ dieser Gleichungen sei kleiner als $m n$, aber nicht kleiner als $n$, so dass wir schreiben köunen:

$$
N=\mu n+\nu \quad(0 \leqq \nu \leqq n-1 ; \quad 1 \leqq \mu \leqq m-1),
$$

Es werde nun angenommen, dass die Gleichungen (A) sich in folgender Weise auflösen lassen:

(A)

$$
\left\{\begin{array}{c}
-p_{1}^{1}+\varphi_{1}^{1}=0,-p_{2}^{1}+\varphi_{2}^{1}=0 \cdot-p_{\mu}^{1}+p_{\mu}^{1}=0,-p_{\mu+1}^{1}+\varphi_{\mu+1}^{1}=0 \\
-p_{1}^{2}+\varphi_{1}^{2}=0,-p_{2}^{2}+\varphi_{2}^{2}=0 \cdot-p_{\mu}^{2}+p_{\mu}^{2}=0, \\
\cdot \cdot \cdot p_{\mu+1}^{2}+\varphi_{\mu+1}^{v}=0 \\
-p_{1}^{n}+\varphi_{1}^{n}=0,-p_{2}^{n}+\varphi_{2}^{n}=0 \cdot-p_{\mu}^{n}+p_{\mu}^{n}=0,
\end{array}\right.
$$

Hierin bedeuten die $\varphi_{a}^{b}$ gewisse Functionen der Variabeln $x_{i}$, $z^{k}$, $p_{\mu+1}^{\nu+1}, p_{\mu+1}^{n}, p_{\mu+2}^{1} \cdots p_{m}^{n}$. Der von den Herrn Méray und Riquier ${ }^{* *}$ ) eingeführten Terminologie folgend nennen wir die $N$ Grössen $p_{1}^{1} \cdots p_{1}^{n}$, $p_{2}^{ \pm} \ldots p_{\mu}^{n}, p_{\mu+1}^{1} \ldots p_{\mu+1}^{v}$, principate ${ }^{4 t}$, die übrigen $m n-N$ Grössen $p_{i}^{k}$ "parametrische" Ableitungen 1. 0 ; die ersteren werden im Folgenden generell mit $p_{a}^{b}$, die letzteren mit $p_{g}^{h}$ bezeichnet. Dementsprechend

" Diese Theorie findet sich skizzirt in meiner Note: "Sur l'intégration etc.", Comptes Rendus rom 3. Angust 1896.

*) Ann. de l'Ec, Norm. (3) VII (1890). 
theilen wir die $m n$ Indicespaare $(i, k)(i=1 \ldots m, k=1 \ldots n)$ in zwei Classen, indem wir die $N$ Paare $(a, b)$ der ersten, die Paare $(g, h)$ der zweiten zuzählen; endlich werde die zweite Ableitung

$$
r_{i j}^{k} \equiv \frac{\partial^{2} z^{k}}{\partial x_{i} \partial x_{j}}
$$

"parametrisch" oder "einfach prineipal" oder „doppelt principal" genannt, je nachdem von den beiden Zahlenpaaren $(i, k)(j, k)$ keines oder eines oder alle beide der ersten Classe angehören.

2. Indem wir die Gleichungen (A') partiell nach $x_{\mu+2} \ldots x_{m}$ differentiiren, wobei $z^{i}, p_{i}^{k}$ als Functionen der $x$ zu betrachten sind, erhalten wir die einfach principalen Ableitungen

$$
r_{a, \mu+2}^{b}, r_{a, \mu+3}^{b} \cdots r_{a, m}^{b}
$$

ausgedrückt als ganze lineare Functionen $\psi_{\alpha, \mu+2}^{b} \cdots \psi_{a, m}^{b}$ der parametrischen 2. Ableitungen, deren Coefficienten von den Variabeln $x, z, p_{g}^{h}$ abhängen. Durch Differentiation der Gleichungen

$$
-p_{\alpha}^{\beta}+\varphi_{\alpha}^{\beta}=0(\beta=\nu+1 \ldots n, \alpha=1 \ldots \mu)
$$

nach $x_{\mu+1}$ gewinnen wir für die noch übrigen einfach principalen $A \cdot b-$ leitungen $r_{\alpha_{1}, \mu+1}^{\beta}$ Ausdrücke, die ausser von parametrischen Derivirten noch von einfach principalen Ableitungen

$$
r_{s, \mu+1}^{t}(s=\mu+2 \ldots m, t=1 \ldots v)
$$

abhängen; ersetzen wir diese Grössen durch ihre vorhin gefundenen Werthe $\psi_{\mu+1, a}^{t}$, so sind schliesslich alle einfach principalen 2. Ableitungen $r_{a l}^{b}$ dargestellt durch ganze lineare Ansdrücke $\psi_{a l}^{b}$ in den parametrischen 2. Ableitungen; die Coefficienten der letzteren sind Functionen von $x, z, p_{g}^{\prime 2}$.

3. Sind $(a, b)\left(a^{\prime}, b\right)$ Zahlenpaare der ersten Classe, so gewinnt man durch partielle Differentiation der Gleichung:

$$
-p_{a}^{b}+\varphi_{a}^{b}=0
$$

nach $x_{a^{\prime}}$ für die doppelt principale Ableitung $r_{a_{a^{\prime}}}^{b}$ einen Ausdruck, der zunächst noch von den parametrischen und einfach principalen 2. Derivirten abhängt, aber, indem man letztere durch ihre obigen Werthe ersetzt, in eine Linearfunction $\psi_{a \alpha}^{b}$ der parametrischen $r_{i j}^{k}$ übergeht. Ein ähnlicher Ausdruck $\psi_{\alpha^{\prime} a}^{b}$ ergibt sich für $r_{a a^{\prime}}^{b}$ ans der Gleichung

$$
-p_{a^{\prime}}^{b}+\varphi_{a^{\prime}}^{b}=0
$$

dureh Differentiation nach $x_{a}$. Indem wir verlangen, dass die Identitäten

$$
\psi_{\alpha \alpha^{\prime}}^{b} \equiv \psi_{a^{\prime} \alpha}^{b}
$$

keine Relation zwischen den parametrischen Ableitungen begründen, d. h. unabhängig von den Werthen derselben erfüllt seien, erbalten 
wir das nachstehende System ron Bedingungsgleichungen, worin zar Abkürzung:

$$
\begin{aligned}
& P_{a g}^{b h} \equiv \frac{\partial \varphi_{a}^{b}}{\partial p_{g}^{h}}, \quad K_{a a^{\prime} r}^{b t} \equiv \sum_{\nu+1}^{n} P_{a, \mu+1}^{b \hbar} P_{a^{\prime} r}^{h t}\left(\begin{array}{l}
r=\mu+2 \ldots m \\
t=1 \ldots \nu
\end{array}\right) \\
&\left.\mathcal{M}_{a l}^{b} \equiv \frac{\partial \varphi_{a}^{b}}{\partial x_{l}}+\sum_{I}^{n} p_{l}^{h} \frac{\partial \varphi_{a}^{b}}{\partial z^{h}} *\right)
\end{aligned}
$$

gesetzt ist:

(B)

$$
\begin{aligned}
& \int(1) \sum_{1}^{v}\left[\left(K_{a a^{\prime} r}^{b t}-K_{a^{\prime} a r}^{b t}\right) P_{\mu+1, s}^{t z}+\left(K_{a a^{\prime} s}^{b t}-K_{a^{\prime} a s}^{b t}\right) P_{\mu+1, r}^{t z}\right] \\
& \equiv \sum_{1}^{n h}\left(P_{a s}^{k l} P_{a^{\prime} r}^{b h}-P_{a^{\prime} s}^{h l} P_{a r}^{b h}+P_{a r}^{h l} P_{a^{\prime} s}^{b h}-P_{a^{\prime} r}^{h l} P_{a s}^{b h}\right) \\
& \text { (2) } \sum_{1}^{\nu}\left(K_{a a^{\prime} r}^{b t}-K_{a^{\prime} a r}^{b t}\right) P_{\mu+1, \mu+1}^{t j}+\sum_{i+1}^{n}\left(P_{a^{\prime} r}^{h j} P_{a, \mu+1}^{b h}-P_{a, r}^{h j} P_{a^{\prime}, \mu+1}^{b h}\right) \\
& \equiv \sum_{1}^{n}\left(P_{a r r}^{b h} P_{a, \mu+1}^{h j}-P_{a r}^{b \hbar} P_{a^{\prime}, \mu+1}^{h j}\right) \\
& \text { (3) } \sum_{n+1}^{n}\left(P_{a_{1}, \mu+1}^{b \hbar} P_{a^{\prime}, \mu+1}^{k j}-P_{a^{\prime}, \mu+1}^{b \hbar} P_{a, \mu+1}^{h j}\right)=0 \text {, }
\end{aligned}
$$

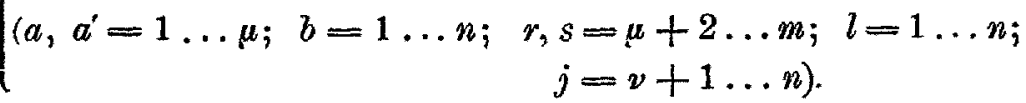

$$
\begin{gathered}
M_{a a^{\prime}}^{b}-M_{a^{\prime} a}^{b}+\sum_{g, h}\left(P_{a g}^{b h} M_{a^{\prime} g}^{h}-P_{a^{\prime} g}^{b h} M_{a g}^{h}\right) \\
\equiv \sum_{\mu+2}^{m} \sum_{1}^{\nu}\left(K_{a^{\prime} a s}^{b t}-K_{a d^{\prime} s}^{b t}\right) M_{\mu+1, s}^{t} \\
\left(a, a^{\prime}=1 \ldots \mu ; b=1 \ldots n\right)
\end{gathered}
$$$$
\text { (C) }\left\{\begin{array}{l}
(1) \sum_{1}^{v}\left[\left(P_{a r}^{b t}-K_{\mu+1, a r}^{b t}\right) P_{\mu+1, s}^{t l}+\left(P_{a s}^{b t}-K_{\mu+1, a s}^{b t}\right) P_{\mu+1, r}^{t l}\right] \\
\equiv \sum_{1}^{n}\left(P_{\mu+1, r}^{b h} P_{a s}^{h l}+P_{\mu+1, s}^{b h} P_{a r}^{h l}\right),
\end{array}\right.
$$

*) Für die principalen 1, Ableitungen sind bierin ihre Werthe ans (A') za sobstituiren. 


$$
\begin{aligned}
& \left\{(2) P_{a r}^{b j}+\sum_{1}^{t}\left(P_{a r}^{b t}-K_{\mu+1, a r}^{b t}\right) P_{\mu+1, \mu+1}^{t j}\right. \\
& \text { (C) }\left\{\sum_{\nu+1}^{n} P_{a r}^{h j} P_{\mu+1, \mu+1}^{b h}+\sum_{1}^{n} P_{k+1, r}^{b h} P_{a, \mu+1}^{n j}\right. \text {, } \\
& \left\{\begin{array}{l}
(3) P_{a, \mu+1}^{b j}=\sum_{\nu+1}^{n} P_{\alpha_{s} \mu+1}^{h j} P_{\mu+1, \mu+1}^{b h}, \\
(a=1 \ldots \mu ; b=1 \ldots \nu ; l=1 \ldots n ; r, s=\mu+2 \ldots m ; \\
j=v+1 \ldots n),
\end{array}\right. \\
& M_{\mu+1, a}^{b}-M_{a, \mu+1}^{b}+\sum_{g h} P_{\mu+1, g}^{b \bar{h}} M_{a g}^{b} \\
& \equiv \sum_{\mu+2}^{m} \sum_{1}^{v}\left(P_{a s}^{b t}-K_{\mu+1, a s}^{b t}\right) M_{\mu+1, s}^{t} \quad(a=1 \ldots \mu ; b=1 \ldots v) .
\end{aligned}
$$

Für $\mu=1$ kommen die Relationen (B) (B'), für $\nu=0(C)\left(C^{\prime}\right)$ in Wegfall; ist $\mu=1, \nu=0$, so bestehen überhaupt keine Relationen für die partiellen Ableitungen der $\varphi_{a}^{b}$; ist $\mu=m-1$ oder $\nu=0$, so verschwinden alle von den Grössen $\boldsymbol{K}_{a a^{\prime} s}^{b t}$ abhängenden Terme.

Sind die Bedingungen (B) $\left(B^{\prime}\right),(C)\left(C^{\prime}\right)$, wie wir fortab voraussetzen, identisch, d. h. für beliebige Werthe der $x, z, p_{g}^{h}$ erfüllt, so werde (A) ein Involutionssystem genannt, und zur Abkürzung mit $J$ bezeichnet.

4. Ueberträgt man die Benennungen ,parametrisch", „einfach principal "s u. s. W. auf die dritten Ableitungen $r_{i j l}^{k}$ der $z^{k}$, so erkennt man leicht, dass nunmehr vermöge der Gleichungen, die aus $\left(A^{\prime}\right)$ durch zweimalige partielle Ableitung nach den $x$ entstehen, sich auch für jede einfach, zweifach und dreifach principale 3. Ableitung $r_{i j l}^{k}$ je ein und nur ein in den parametrischen 3. Ableitungen linearer Ausdruck ergiebt, dessen Coefficienten von $x, z$, und den parametrischen 1. und 2. Ableitangen abhängen; Analoges gilt für die Derivierten beliebiger Ordnung*).

5. Die Relationen (C) (C') zeigen, dass jedes einzelne der $\mu$ Systeme von Gleichungen

$$
\left\{\begin{array}{l}
-p_{\alpha}^{1}+\varphi_{\alpha}^{1}=0, \ldots,-p_{\alpha}^{n}+\varphi_{\alpha}^{n}=0 \\
-p_{\mu+1}^{1}+\varphi_{\mu+1}^{1}=0, \ldots,-p_{\mu+1}^{v}+\varphi_{\mu+1}^{v}=0
\end{array}\right.
$$

für sich genommen ein Involutionssystem bildet, wenn man darin die Grössen $x_{1} \ldots x_{\alpha-1}, x_{\alpha+1} \ldots x_{\mu}$ als constante Parameter betrachtet; die Gleichungen (2) mögen als "Theilsystem $J_{\alpha}$ " bezeichnet werden. 
Sind ferner $\alpha_{1} \ldots \alpha_{s}$ irgend welche Zablen der Reihe $1,2 \ldots \mu$, so bilden die $s n+\nu$ Gleichungen, aus welehen die Theilsysteme $J_{\alpha_{1}}, J_{\alpha_{2}}, \ldots, J_{\alpha_{s}}$ bestehen, ebenfalls ein Involutionssystem, wenn man die ausser $x_{\alpha_{1}} \ldots x_{\alpha_{s}}$ noch vorhandenen Grössen der Reihe $x_{1} \ldots x_{\mu}$ als Parameter ansieht.

6. Für die unaufgelöste Form (A) von $J$ lassen sich die Bedingungen der Nr. 3 folgendermassen formuliren: durch einmalige partielle Differentiation von (A) nach $x_{1} \ldots x_{m}$ erhält man $m \mathrm{~N}$ in den $\frac{1}{2} m(m+1) n$ Unbekannten $r_{i l}^{k}$ lineare Gleichungen:

(3) $f_{i l} \equiv M_{i l}+\sum_{1}^{n} \sum_{1}^{m} \mathrm{P}_{i j}^{k} r_{j l}^{k}=0 ; i=1 \ldots N_{i} l=1 \ldots m$, worin

$$
\mathrm{M}_{i l} \equiv \frac{\partial f_{i}}{\partial x_{l}}+\sum_{1}^{n} \frac{\partial f_{i}}{\partial z^{k}} p_{l}^{k}, \quad \mathrm{P}_{i j}^{k} \equiv \frac{\partial f_{i}}{\partial p_{j}^{k}}
$$

gesetzt ist. Diese Gleichungen sollen nun alle parametrischen $A b-$ leitangen $r_{j l}^{k}$ willkürlich lassen; die Anzahl derselben ist

$$
(m-\mu)(n-\nu)+\frac{1}{2} n(m-\mu)(m-\mu-1),
$$

mithin sind genau

$$
\mathrm{N}^{\prime}=\mu \nu+\frac{1}{2} n \mu(\mu-1)
$$

der Relationen (3) eine Consequenz der übrigen, d. h. es verschwinden alle $\left(m N-N^{\prime}+1\right)$ reihigen, aber nicht alle $\left(m N-N^{\prime}\right)$ reihigen Determinanten der zu dem Gleichnngssystem (3) gehörigen Matrix, die aus $m \mathrm{~N}$ Zeilen und $\frac{1}{2} n(m+1) m+1$ Colonnen besteht*).

Offenbar sind auch umgekehrt die so erhaltenen Bedingungsgleichungen mit denjenigen der Nr. 3 völlig äquivalent.

7. Wir stellen in dieser $\mathrm{Nr}$. einige der von Herm Bourlet veröffentliehten Resultate und Bezeichnungen zusammen ${ }^{* *}$ ).

Jedes System von linearen partiellen Differentialgleichungen in $m$ unabhängigen Variabeln $x_{1} \ldots x_{m}$ und $p$ unbekannten Functionen $u_{1} \ldots u_{p}$ kann durch Auflösung nach einer gewissen Zahl von Ableitungen $\frac{\partial u_{i}}{\partial x_{k}}$ auf eine Form gebracht werden, in der es nur Gleichungen der folgenden Art enthält:

*) Dies folgt auch daraus, dass $\mathrm{N}^{\prime}$ die Anzahl der verschiedenen Identitãten (1) ist.

*) Vgl die Anmerkung pag. 547. 


$$
\frac{\partial u_{i}}{\partial x_{k}}=a_{00}^{i k}+\sum_{i+1}^{p} a_{j k}^{i k} \frac{\partial u_{j}}{\partial x_{k}}+\sum_{1}^{p} \sum_{k+1}^{m} a_{j k}^{i k} \frac{\partial u_{j}}{\partial x_{k}} ;
$$

die Coefficienten $a_{r s}^{i k}$ sind Functionen der $x$ und $u$.

Ein solches System heisst canonisch; die Variable $x_{k}$ heisst „principal“ oder ,parametrisch" in Bezug auf $u_{i}$, je nachdem $\frac{\partial u_{i}}{\partial x_{k}}$ auf der linken Seite einer der Gleichungen (4) auftritt oder nicht, und entsprechend wird anch die Ableitung $\frac{\partial u_{i}}{\partial x_{k}}$ principal oder parametrisch genannt; eine zweite Ableitung $\frac{\partial^{2} u_{i}}{\partial x_{k} \partial u_{l}}$ wird parametrisch, einfach oder doppelt principal genannt, je nachdem keine, oder eine oder alle beide Variabeln $x_{k}, x_{l}$ in Bezug anf $u_{i}$ principal sind. Die einfach principalen 2. Ableitungen können mit Hülfe der Gleichungen, die aus (3) durch partielle Differentiation nach den $x$ folgen, als Linearfunctionen der parametrischen 2. Ableitungen dargestellt werden, deren Coeffcienten von den $x, u$ und von parametrischen 1, Ableitungen abhängen. Für die doppelt principalen Derivirten erhält man dagegen im allgemeinen zwei verschiedene derartige Darstellungen; sind diese für alle Werthe $\operatorname{der} x, u$ and der 1. und 2. parametrischen Ableitungen identisch, so heisst das canonische System (4) unbeschränkt integrabel.

Sind die Coefficienten $\alpha_{r s}^{i z}$ in der Umgebung der Stelle $x_{h}=x_{h}^{0}$, $u_{k}=u_{k}^{0}$ holomorph, und ist $\varphi_{i}$ eine arbiträre Function der in Bezug auf $u_{i}$ parametrischen Variabeln $x_{k}$, die an der Stelle $x_{j}=x_{j}{ }^{0}$ holomorph ist und den Werth $u_{i}^{0}$ annimmt, so giebt es ein und nur ein System von Functionen $u_{i}$ der Variabeln $x_{h}$, die an der Stelle $x_{k}=x_{h}^{0}$ holomorph sind, dem unbeschränkt integrabeln System (4) identisch genügen, und sich resp. auf $\varphi_{i}$ reduciren, wenn die in Bezug auf $u_{i}$ prineipalen Variabeln $x_{l}$ die Werthe $x_{l}^{0}$ annehmen.

8. Indem wir za den Bezeichnungen der Nr. 3 zurückkehren, betrachten wir das folgende System linearer partieller Differentialgleichungen 1. 0 .:

$$
\begin{cases}\frac{\partial p_{a}^{b}}{\partial x_{a^{\prime}}}=\psi_{a a^{\prime}}^{b}, & \frac{\partial p_{a^{\prime}}^{b}}{\partial x_{a}}=\psi_{a a^{\prime}}^{b}, \\ \frac{\partial p_{a}^{b}}{\partial x_{i}}=\psi_{a b}^{b}, & \frac{\partial p_{l}^{b}}{\partial x_{a}}=\psi_{a b}^{b}, \\ \frac{\partial p_{g}^{b}}{\partial x_{g^{\prime}}}=\frac{\partial p_{g^{\prime}}^{h^{\prime}}}{\partial x_{g}}, & \frac{\partial z^{k}}{\partial x_{i}}=p_{i}^{z}, \quad(i=1 \ldots m ; k=1 \ldots n)\end{cases}
$$

In diesen Gleichungen durehlaufen die Zahlenpaare $(a, b)\left(a^{\prime} b\right)$ alle Paare der 1. Classe; für jedes einzelne Werthsystem $(a, b)$ ist 
ferner $l$ auf alle mögliche Arten sa zu wählen, dass $(l, b)$ der 2 . Classe angehört. Die Zahlenpaare $(g, h)(g, h)$ durchlanfen alle Paare der 2. Classe, und zwar so, dass stets $g^{\prime}<g$; in den Ausdrücken $\varphi_{i j}^{k}$ endlich sind die parametrischen Ableitungen in der Form $\frac{\partial p_{g}^{b}}{\partial x_{g}}$ zu schreiben, wo $g \leqq g$.

Bezeichnen wir nun die Grössen

$$
z^{1} \ldots z^{n}, p_{1}^{1} \ldots p_{1}^{n}, p_{2}^{1} \ldots p_{m}^{n}
$$

in der hier hingeschriebenen Reihenfolge mit $u_{1}, u_{2} \ldots u_{p}(p=n(m+1))$, so geht das System (5) über in ein unbeschränkt integrables canonisches System der Form (4), wie aus den Bemerkungen der Nr. 4 und der Entstehungsweise der Ansdrücke $\psi_{i j}^{k}$ unmittelbar hervorgeht, und zwar sind in Bezug anf die Functionen $z, p_{\alpha}^{b}$ alle Variabeln $x$ principal, dagegen :

für $p_{\mu+1}^{v+1} \ldots p_{\mu+1}^{n}: x_{1} \ldots x_{\mu}$ principal, $x_{\mu+1} \ldots x_{m}$ parametrisch;

$$
\begin{aligned}
& " p_{\mu+2}^{1} \cdots p_{\mu+2}^{\mathrm{z}}: x_{1} \ldots x_{\mu+1} \quad ", x_{\mu+2} \cdots x_{m n} \quad " \quad \text {; } \\
& \text { " } p_{m}^{1} \quad \ldots p_{m}^{n}: x_{1} \ldots x_{m-1} \quad, \quad, x_{m}
\end{aligned}
$$

Wir verstehen nun unter $\bar{x}_{i}, \bar{z}^{k}, \bar{p}_{i}^{k} n(m+1)+m$ willkürliche Constante, ferner für jedes Zahlenpaar $(g, h)$ der II. Classe unter $\pi_{g}^{h}$ eine arbiträre Function der Variabeln $x_{g}, x_{g+1} \ldots x_{m}$, die sich an der Stelle $x_{i}=\bar{x}_{i}$ holomorph verhält und auf die Constante $\bar{p}_{g}^{h}$ reducirt; sind nun die Functionen $\varphi_{a}^{b}$ in der Umgebung der Stelle $\bar{x}, \bar{z}, \bar{p}_{g}^{k}$ holomorph, so existirt nach Nr. 7 ein und nur ein den Gleichungen (5) identisch genügendes System von Functionen $z^{i}, p_{i}^{k}$ von der Beschaffenheit, dass $z^{i}, p_{a}^{b}$ für $x_{1}=\bar{x}_{1} \ldots x_{m}=\bar{x}_{m}$ bez. die Werthe $\bar{z}^{i}, \bar{p}_{a}^{b}$ annehmen, während sich die Grössen $p_{g}^{h}$ für $x_{t}=\bar{x}_{1} \ldots x_{g-1}=\bar{x}_{g-1}$ bez. auf die arbiträren funktionen $\pi_{g}^{h}$ reduciren. Substituiren wir die so definirten Functionen $z^{i}, p_{i}^{\bar{k}}$ in die Gleichungen (A'), so gehen die linken Seiten derselben über in Functionen von $x_{1} \ldots x_{n}$, deren $A b$ leitungen wegen (5) identisch verschwinden, d. h. in Constante; die vorhin erbaltenen Functionen $z^{1} \ldots z^{n}$ sind also Integrale des gegebenen Systems $J$, wenn die Integrationsconstanten den Bedingungen

$$
-\bar{p}_{a}^{b}+\varphi_{a}^{b}\left(\bar{x}, \bar{z}, \bar{x}_{y}^{\natural}\right)=0
$$

unterworfen werden.

9. Man kann mit Hülfe von Quadraturen $v$ Functionen $\xi^{i}(i=1 \ldots v)$, der Variabeln $x_{\mu+2} \ldots x_{m}$ auf eine einzige Weise so bestimnen, dass man identisch hat:

$$
\frac{\partial \xi^{i}}{\partial x_{\mu+2}} \equiv \pi_{\mu+2}^{i}
$$


dass sich ferner die Ableitungen

für

$$
\frac{\partial \xi^{i}}{\partial x_{\mu+s}} \quad(s=3,4 \ldots m-\mu)
$$

$$
x_{\mu+2}=\bar{x}_{\mu+2} \ldots x_{\mu+s-1}=\bar{x}_{\mu+s-1}
$$

bez. auf die arbiträren Functionen $x_{\mu+s}^{i}$ reduciren, nnd überdies $\xi^{i}$ an der Stelle $\bar{x}$ den Werth $\bar{z}^{i}$ annimmt; desgleichen giebt es ein einziges System von Functionen $\xi^{k}(k=\nu+1 \ldots n)$ der Variabeln $x_{\mu+1} \ldots x_{m}$, derart, dass:

ferner die Ableitungen

$$
\frac{\partial \zeta^{k}}{\partial x_{\mu+1}} \equiv \pi_{\mu+1}^{k}
$$

$$
\frac{\partial \xi^{k}}{\partial x_{\mu+s}} \quad(s=2,3 \ldots m-\mu)
$$

für

$$
x_{\mu+1}=\bar{x}_{\mu+1} \ldots x_{\mu+s-1}=\bar{x}_{\mu+s_{-1}}
$$

bez. in die Ausdrücke $\pi_{\mu+s}^{k}$ übergehen, endlich $\xi^{k}$ an der Stelle $\bar{x}$ den Werth $\bar{z}^{k}$ erhält; umgekehrt sind bei willkürlicher Wahl der Functionen $\xi$ die $\pi_{g}^{h}$ durch obige Bedingungen eindentig bestimmt; hieraus ergiebt sich mit Hülfe der vorigen Nr, das folgende Theorem:

"Es seien $\xi^{1} \ldots \xi$ arbiträre Functionen von $x_{\mu+2} \ldots x_{m}$, ferner $\xi^{n+1} \ldots \xi^{n}$ ebensolche von $x_{\mu+1} \ldots x_{m}$; für $x_{i}=\bar{x}_{i}$ gehe $\xi^{i}$ in $\bar{z}^{i}$, $\frac{\partial \xi^{h}}{\partial x_{g}}$ in $\bar{p}_{g}^{h}$ über. Sind dann die Functionen $\varphi_{a}^{b}$ an der Stelle $\bar{x}, \bar{z}, \bar{p}_{g}^{k}$ holomorph, so giebt es ein und nur ein an der Stelle $\bar{x}_{1} \ldots \bar{x}_{m}$ reguläres Integralsystem $z^{1} \ldots z^{*}$ des Involutionssystems $J$, von der Beschaffenheit, dass $z^{1} \ldots z^{*}$ für

$$
x_{1}=\bar{x}_{1} \ldots x_{\mu+1}=\bar{x}_{\mu+1}
$$

sich bez. auf $\xi^{2} \ldots \xi^{2}$, ferner $z^{*+1} \ldots z^{n}$ für

$$
x_{1}=\bar{x}_{1} \ldots x_{\mu}=\bar{x}_{\mu}
$$

bez. auf $\xi^{+1} \ldots \xi^{n}$ reduciren. Wir nennen das so definirte System $z^{1} \ldots g^{n}$ das allgemeine Integral von $J$, in das sonach $n-\nu$ willFürliche Functionen von $m-\mu$ Argumenten, sonoie $\nu$ willkiirliche Functionen von $j e m-\mu-1$ Argumenten eingehen."

10. Ein Werthsystem $z^{1} \ldots z^{n} x_{1} \ldots x_{m} p_{1}^{1} \ldots p_{m}^{n}$ heisst nach Lie ein "Element"; zwei benachbarte Elemente $x+d x, z+d z, p_{i}^{\bar{k}}+d p_{i}^{k}$ heissen "vereinigt liegend", wenn die Relationen

$$
d z^{k}=\sum_{i}^{m} p_{i}^{k} d x_{i}
$$

erfüllt sind. Fine $m-q$-fach ausgedehnte Mannigfaltigkeit von Elementen, die den totalen Differentialgleichungen (6) identisch genïgt, 
heisse eine "Element- $M_{m-q}$ ". In der vorliegenden Arbeit wollen wir ausđrücklich nur solche Elementmannigfaltigkeiten betrachten, welche durch Gleichungen der Form:

$$
\left\{\begin{array}{l}
x_{s}=\xi_{s}\left(x_{q+1}, x_{q+2} \ldots x_{m}\right), \quad(s=1 \ldots q) \\
z^{i}=z^{i}\left(x_{q+1} \ldots x_{m}\right) \\
p_{i}^{k}=p_{i}^{k}\left(x_{q+1} \ldots x_{m}\right)
\end{array}\right.
$$

definirt werden können, deren zugehörige Pnnktmannigfaltigkeit also ebenfalls $m-q \cdot$ fach ausgedehnt ist.

Eine Element- $\boldsymbol{M}_{m-q}$, die den Gleichungen (A) identiseh genügt, heisse eine "Integral- $M_{m-q}$ " von $J$. Unter Beibehaltung der Bezeichnungsweise der vorigen $\mathrm{Nr}$. existirt eine und nur eine Integral$\boldsymbol{M}_{m-\mu}$ von $\boldsymbol{J}$, unter deren Definitionsgleichungen die folgenden enthalten sind:

$$
x_{s}=\bar{x}_{s}(s=1 \ldots \mu) ; \quad z^{k}=\xi^{k}\left(x_{\mu+1} \ldots x_{\omega}\right)(k=\nu+1 \ldots n)
$$

und welche der Bedingang genügt, dass sich die Ausdrücke von $z^{1} \ldots z^{v}$ für $x_{\mu+1}=\bar{x}_{\mu+1}$ bez. auf $\xi^{1}\left(x_{\mu+2} \ldots x_{m}\right), \xi^{2} \ldots \xi$ reduciren. Denn das System ron $\nu$ Gleichungen in den abbängigen Variabeln $z^{1} \ldots z^{\nu}$ und den Independenten $x_{\mu+1} \ldots x_{m}$, das man erhält, wenn man in den Relationen

$$
\frac{\partial z^{i}}{\partial x_{\mu+1}}=\varphi_{\mu+1}^{i} \quad(i=1 \ldots \nu)
$$

für die $x_{s}$ und $z^{z}$ ihre Ausdrücke (8) substituirt, besitzt nach der vor. Nr. ein und nur ein System von Integralen $z^{i}\left(x_{\mu+1} \ldots x_{m}\right)$, das den obigen Festsetzungen entspricht, worauf die noch fehlenden Definitionsgleichungen der gesuchten Integral- $M_{m-\mu}$ sich unmittelbar ans ( $\mathrm{A}^{\prime}$ ) ergeben, wenn man in die Functionen $\varphi_{\alpha}^{b}$ für $x_{1} \ldots x_{\mu} z^{1} \ldots z^{n}$ die erhaltenen Ausdrücke in $x_{\mu+1} \ldots x_{m}$ einsetzt. Ebenso erhält man durch Integration des "Theilsystems $J_{\mu}$ " (vgl. Nr. 5) eine und nur eine Integral- $\boldsymbol{M}_{n-\mu+1}$, welche die soeben bestimmte $\boldsymbol{M}_{m-k}$ umfasst; ferner durch Integration des Systems von Gleichnngen, aus denen die Theilsysteme $J_{\mu-1}, \mathcal{J}_{\mu}$ bestehen, eine ganz bestimmte Integral- $\boldsymbol{M}_{m-\mu+2}$ etc. Man erkennt so, dass durch die zuerst gefundene Integral- $\boldsymbol{M}_{m-\mu}$ successive eine ganz bestimmte Serie von Integralmannigfaltigkeiten $M_{m-\mu+1} \ldots M_{m}$ festgelegt ist, deren jede die vorhergehende ganz in sich enthält.

Der Fall, dass die ersten $\mu$ Definitionsgleichnngen der Ausgangs$\boldsymbol{M}_{m-\mu}$ die allgemeinere Form

$$
x_{s}=\xi_{s}\left(x_{\mu+1} \ldots x_{m}\right) \quad(s=1 \ldots \mu)
$$

besitzen, wird auf den vorhergehenden zurückgeführt, indem man vermôgge dex Gleiehungen: 


$$
\begin{aligned}
& X_{s}-\bar{X}_{s}=x_{s}-\xi_{s}, \quad s=1 \ldots \mu \\
& X_{t}-\bar{X}_{t}=x_{t}-\bar{x}_{t}, \quad t=\mu+1 \ldots m,
\end{aligned}
$$

neue unabhängige Veränderliche $\boldsymbol{X}_{1} \ldots \boldsymbol{X}_{m}$ einführt, wodurch weder die Involutionseigenschaft noch die Gestalt der Gleichungen (A') geändert wird.

Die allgemeinste Integral- $M_{m-\mu}$ der Form (7) ergiebt sich somit durch Integration eines Hiilfssystems von $v$ Gleichungen in $v$ abhängigen und $m-\mu$ unabhängigen Variabeln, im Falle $\nu=0$ also ohne jede Integration; die allgemeinste Integral- $M_{m-\mu+s}$ von $J$ durch Integration eines Involutionssystems, bestehend aus $\mathrm{N}-s n$ Gleichungen in $n a b$ hängigen und $m-\mu+s$ unabhängigen Veränderlichen.

11. Führt man in das System (A) vermöge der Mayer'schen Traasformationsformeln:

$$
\begin{aligned}
& x_{2}=\bar{x}_{2}+y_{1} y_{2} \ldots x_{\mu}=\bar{x}_{\mu}+y_{1} y_{\mu}, \\
& x_{1}=\bar{x}_{1}+y_{1}, x_{\mu+1}=\bar{x}_{\mu+1}+y_{\mu+1} \ldots x_{m}=\bar{x}_{m}+y_{m}
\end{aligned}
$$

neue Independente $y_{1} \ldots y_{m}$ ein, and wird:

$$
\frac{\partial z^{\bar{k}}}{\partial y_{i}}=q_{i}^{\bar{k}}, \psi_{a}^{b} \equiv \varphi_{a}^{b}\left(\bar{x}_{1}+y_{1}, \bar{x}_{2}+y_{1} y_{2} \ldots z^{\mathrm{l}}, \ldots z^{n}, p_{g}^{k}\right)
$$

gesetzt, so geht das System $\left(\mathrm{A}^{\prime}\right)$ über in das folgende:

$$
\begin{aligned}
& \left\{\begin{array}{l}
-q_{1}^{b}+\psi_{1}^{b}+y_{2} \psi_{2}^{b}+\cdots y_{\mu} \psi_{\mu}^{b}=0, \quad b=1 \cdots n \\
-q_{\mu+1}^{c}+\psi_{\mu+1}^{c}=0, \quad c=1 \ldots \nu
\end{array}\right. \\
& -q_{k}^{b}+y_{k} \psi_{k}^{b}=0, \quad k=2,3 \ldots \mu ; \quad b=1 \ldots n \text {. }
\end{aligned}
$$

Die $n+\nu$ Gleichungen (A") bilden nun für sich genommen ein Involutionssystem $J_{0}$ in den unabhängigen Variabeln $y_{1}, y_{\mu+1}, y_{\mu+2}, \ldots y_{m}$, und zwar für beliebige Werthe der als Parameter zu betrachtenden Grössen $y_{2} \ldots y_{\mu}$. In der That: die Relationen (C) (C) der Nr, 3 bestehen der Annahme nach für alle Werthe der Variabeln $x ; z, p_{g}^{\hbar}$, also auch, wenn man die $x$ durch ihre Ausdräcke (9), die $p_{o}^{h}$ durch $q_{o}^{h}$ ersetzt. Wir fassen nun nach erfolgter Substitution eine bestimmte der Relationen (C) oder ( $\left.C^{\prime}\right)$ ins Auge, lassen darin a successive die Werthe $1,2 \ldots \mu$ annehmen, multiplieiren die so erhaltenen, den Indices $a=2,3 \ldots \mu$ entsprechenden Gleichnngen bez. mit $y_{2} \ldots y_{\mu}$ and addiren sie zu der dem Index $a=1$ entsprechenden Gleichung; verfahren wir analog mit allen Beziehungen (C) (C'), so erhalten wir gerade die Bedingungen dafür, dass die Gleichungen ( $A^{\prime \prime}$ ) ein Involntionssystem darstellen. Nach Nr. 3 besitzen dieselben also ein und nur ein Integral $z_{0}{ }^{1}\left(y_{1} \ldots y_{m}\right) \ldots z_{0}^{n}\left(y_{1} \ldots y_{m}\right)$ von der Beschaffenheit, dass $z_{0}^{*}(s=1 \ldots, v)$ für $y_{1}=0, y_{\mu+1}=0$ in den arbiträren 
Ausdruck $\xi^{s}\left(\bar{x}_{\mu+2}+y_{\mu+2}, \ldots \bar{x}_{m}+y_{m}\right)$ übergeht, während sich die Functionen $z_{0}^{t}(t=\nu+1 \ldots n)$ für $y_{1}=0$ bezw. auf die willkürlichen Functionen $\xi^{t}\left(\bar{x}_{\mu+1}+y_{\mu+1} \ldots \bar{x}_{m}+y_{m}\right)$ reduciren. Frsetzt man in den $z_{0}{ }^{i}$, welche die Parameter $y_{2} \ldots y_{\mu}$ augenscheinlich nur in den Verbindungen $y_{1} y_{2}, \ldots y_{1} y_{\mu}$ enthalten, die $y_{6}$ durch ihre aus (9) folgenden Ausdrücke, so erhält man gerade wieder die in Nr. 9 definirten Integralfunctionen $z^{1} \ldots z^{n}$ des gegebenen Systems $J$.

Die Integration von $J$ Jann sonach auf diejenige eines Involutionssystems $J_{0}$ von $n+\nu$ Gleichungen in $n$ abhängigen und $m-\mu+1$ unabhängigen Variabeln zurückgeführt soerden.

\section{Capitel II.}

\section{Die charakteristische Matrix.}

12. Indem wir die Bezeichnungen und Voraussetzungen des vorigen Capitels beibehalten, schreiben wir

$$
L_{i}^{k} \equiv \sum_{1}^{m} s P_{i s}^{k} \lambda_{s} \quad \text { (vgl. Nr. 6), }
$$

worin $\lambda_{1} \ldots \lambda_{m}$ unbestimmte Grössen bedeuten. Das rechteckige Schema

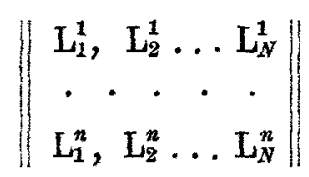

werde die „charalteristische Matrix" des Involutionssystems $J$ genannt. Wir wollen nunmehr folgendes Theorem beweisen:

„Bedeuten die Grössen $x, z, p_{i}^{k}$ irgend welche den Relationen (A) genügende Constanten, und interpretirt man die Variabeln $\lambda_{1}, \lambda_{2} \ldots \lambda_{m}$ als homogene Punticoordinaten eines $m-1$-fach ausgedehnten Raums, so stellen die Gleichungen, die mas durch Nullsetzen aller n-reihigen Determinanten der charalteristischen Matrix (D) erhält, eine $m-\mu-1$ fach ausgedehnte Punktmannigfaltigkeit $\mathrm{M}$ der Ordnung $n-v$ dar", mit andern Worten: „Zu jedem beliebig gewählten System von Werthen $\lambda_{\mu+1}, \lambda_{\mu+2} \ldots \lambda_{m}$ giebt es $n-\nu$ Werthsysteme der Variabeln $\lambda_{1} \ldots \lambda_{\mu}$, volche alle n-reihigen Determinanten von (D) anmulliven".

13. Für die aufgelöste Form (A') von $J$ hat die charakteristische Matrix folgende Gestalt

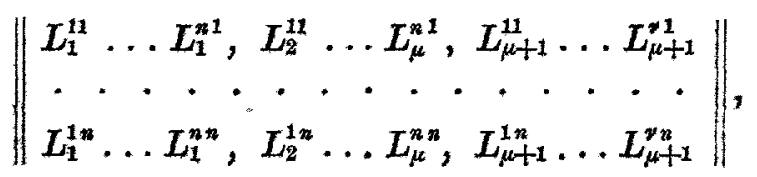


worin gesetzt ist:

$$
\begin{gathered}
L_{a}^{b j} \equiv \lambda_{a} \delta_{b j}+\sum_{\mu+2}^{m} \lambda_{s} P_{a s}^{b j}, j=1,2 \ldots \nu, \\
L_{a}^{b h} \equiv \lambda_{a} \delta_{b h}+\sum_{\mu+2}^{m} \lambda_{s} P_{a s}^{l h}, \quad h=v+1 \ldots n \\
\left(\delta_{i k}=0, \delta_{i i}=-1\right) .
\end{gathered}
$$

Substituiren wir nun in die Relationen (A) für die $p_{a}^{b}$ ihre aus (A') folgenden Werthe und differentiiren die erhaltenen Identitäten nach $p_{g}^{h}$, so kommt:

woraus sofort folgt:

$$
\sum_{a_{s} b} P_{i a}^{b} P_{a g}^{b \hbar}+P_{i g}^{h}=0
$$

$$
\mathrm{L}_{i}^{k} \equiv \sum_{a, b} \mathrm{P}_{i a}^{b} L_{a}^{b k} \quad(i=1 \ldots N, k=1 \ldots n) .
$$

Umgekehrt lassen sich die $n N$ Grössen $L_{a}^{b \bar{k}}$ linear durch die $L_{i}^{k}$ darstellen, da die $N$-reihige Determinante:

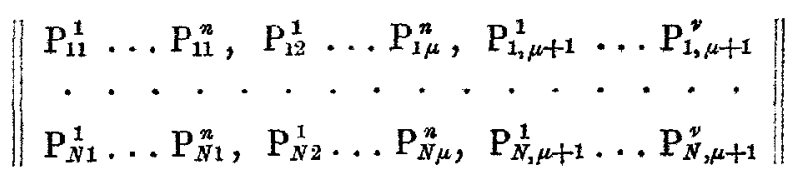

der Annahme nach vermöge (A) nicht Null ist. Die n-reihigen Determinanten der Matrices (D) (D') verschwinden also bez. für dieselben Werthe der $\lambda_{s}$, wenn das Element $x, z, p_{i}^{k}$ innerhalb der durch (A) definirten Mannigfaltigkeit beliebig gewählt ist, and es genügt somit, das Theorem der vorigen Nr. für die Matrix (D') zu erweisen.

14. Wir betrachten die folgenden beiden Matrices, von denen die erste einen Bestandtheil ron $\left(D^{\prime}\right)$ bildet:

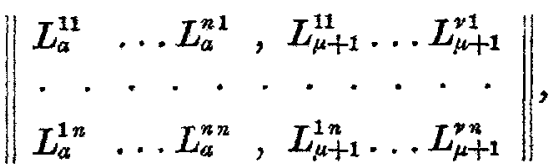

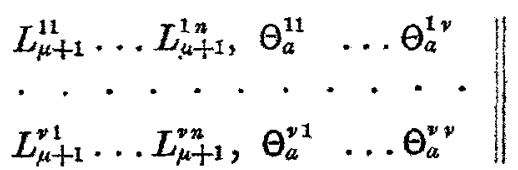

hierin ist

$$
\theta_{a}^{i k} \equiv-L_{a}^{i k}+\sum_{\mu+2}^{m} \lambda_{s} K_{\mu+L_{s} a s}^{i k}
$$


gesetzt, die Grössen $K_{\mu+1, a s}^{i \grave{k}}$ haben die in Nr. 3 erklärte Bedeutung; unter $a$ ist eine beliebige Zahl der Reihe $1 \ldots \mu$ za verstehen. Die Relationen (C) der Nr. 3 drücken aus, dass für beliebige Werthe der $\lambda_{i}$ und für alle Indices $h=1,2, \ldots n ; l=1,2, \ldots \nu$ die folgende Identität besteht:

$$
\sum_{1}^{n} L_{a}^{s h} L_{\mu+1}^{l \alpha}+\sum_{1}^{\nu} L_{\mu+1}^{t \hbar} \Theta_{a}^{b t} \equiv 0
$$

d. h. also, dass die beiden Matrices $(\alpha)(\beta)$ für jedes Werthsystem der $\lambda$ einander correspondiren *). Eine beliebige $n$-reihige Determinante des Schema's ( $\alpha$ ) ist demnach bis auf das Zeichen identisch gleich dem Product einer Function $K_{\alpha}$ der Variabeln $\lambda$ in die complementäre $\nu$-reihige Determinante von $(\boldsymbol{\beta})$. Aber die ganz-rationalen homogenen Functionen der $\lambda$, welche durch die $\nu$-reihigen Determinanten von $(\beta)$ dargestellt werden, besitzen keinen gemeinsamen, von den $\lambda_{i}$ abhängigen Factor, da die beiden Determinanten, welche aus den ersten, resp. letzten $\nu$ Colonnen von $(\beta)$ bestehen, von $\lambda_{a}$ bezw. $\lambda_{\mu+1}$ frei sind, und resp. die Glieder $\left(-\lambda_{\mu+1}\right)^{\nu},\left(-\lambda_{a}\right)^{y}$ enthalten. Der gemeinschaftliche Factor $K_{a}$ aller n-reihigen Determinanten von $(\alpha)$ ist demnach ein ganzrationaler homogener Ausdruck $n-v$. Grades in $\lambda_{\alpha}, \lambda_{\mu+1}, \lambda_{\mu+2} \ldots \lambda_{m}$, der angenscheinlich das Glied $\left(-\lambda_{a}\right)^{2-1}$ enthält, und dessen Coeffieienten in den Grössen $\boldsymbol{P}_{a g}^{b h}$ rational sind.

15. Dureh die erhaltenen Resultate ist unser Theorem im Falle $\mu=1$ bereits bewiesen. In diesem Falle nämlich ist das Verschwinden aller $n$-reihigen Determinanten von $(\alpha)$ äquivalent mit einer einzigen ganzrationalen homogenen Gleichung $n-\nu$. Grades in $\lambda_{1} \lambda_{2} \ldots \lambda_{m}$ :

$$
K\left(\lambda_{1}, \lambda_{2} \ldots \lambda_{m}\right)=0 \text {. }
$$

Wir wollen die Form $K$ gelegentlich als die "charahteristische Form" des Involutionssystems $J(\mu=1)$ bezeichnen.

Diese Thatsache lässt sich auch unmittelbar aus den Bemerkungen der Nr. 6 erschliessen. Darnach existiren nämlich $N^{\prime}=v$ linear unabhängige Systeme von je $m N$ Functionen $\alpha_{i l}^{s}(s=1 \ldots \nu)$ der Grössen $x, z, p_{i}^{k}$ von der Beschaffenheit, dass für beliebige Werthe der zweiten Ableitungen $r_{i j}^{k}$ die Identitäten stattfinden:

$$
\sum_{1}^{N} \sum_{1}^{j n} \alpha_{i l}^{s} f_{i l}=0 \quad(s=1 \ldots \nu)
$$

\section{Hierans folgen die Beziehungen:}

*) Vgl. z. B. Gordam-Kerschensteiner, Vorlesungen über Invariantentheorie I Leipzig 1885. pag. 94ff. 


$$
\begin{gathered}
\sum_{1}^{N} \sum_{1}^{m} \alpha_{i l}^{s} M_{i l} \equiv 0, \quad \sum_{i}^{N} \alpha_{i j}^{s} P_{i l}^{k}+\sum_{1}^{N} \alpha_{i l}^{s} P_{i j}^{k} \equiv 0 \\
(l, j=1 \ldots m ; k=1 \ldots n) .
\end{gathered}
$$

Setzt man demnach

$$
\pi_{i}^{s} \equiv \lambda_{1} \alpha_{i 1}^{s}+\lambda_{2} \alpha_{i 2}^{s}+\cdots+\lambda_{m} \alpha_{i m}^{s},
$$

so hat man für $s=1 \ldots v, k=1 \ldots n$ die Identität

$$
\sum \pi_{i}^{s} L_{i}^{k} \equiv 0
$$

d. h. die Matrix der Ausdrücke $\pi_{i}^{s}$ ist zu (D) correspondirend, woraus das Theorem der Nr. 12 für den Fall $\mu=1$ wie oben gefolgert wird.

16. Wir wenden uns nun zu dem allgemeinen Fall $\mu>1$. Da sich unter den Definitionsgleichungen der im Theorem genannten Mannigfaltigkeit $M$ die $\boldsymbol{\mu}$ Gleichungen

$$
K_{1}=0, K_{2}=0 \ldots K_{\mu}=0
$$

vorfinden, so ist die Dimensionszahl von $M$ sicher $\leqq m-\mu-1$; erreicht sie diese Zahl und ist $r$ die Ordnung von $\bar{M}$, so dass bei beliebigen Werthen von $\lambda_{\mu+1} \ldots \lambda_{m}$ die ev. theilweise coincidirenden $r$ Werthsysteme $\lambda_{1}^{(s)} \ldots \lambda_{\mu}^{(s)}(s=1 \ldots r)$ alle $n$-reihigen Determinanten von $\left(D^{\prime}\right)$, also auch von (D) annulliren, so ist der in $\operatorname{den} \lambda_{a}, \lambda_{\mu+1} \ldots, \lambda_{m}$ ganzrationale homogene Ausdruck

$$
\prod_{s=1}^{r}\left(-\lambda_{a}+\lambda_{a}^{(s)}\right)
$$

angenscheinlich als Factor in $K_{a}$ enthalten, also ist $r \leqq n-\nu$.

17. Um den Beweis für $\mu>1$ zu Ende zu führen, verstehen wir unter $a^{\prime}$ eine von $a$ verschiedene Zahl der Reihe $1 \ldots \mu$ und setzen:

$$
H_{a a^{\prime}}^{b k} \equiv \sum_{\mu+2}^{m} s \lambda_{s}\left(K_{a a^{*} s}^{b k}-K_{a^{\prime} a s}^{b k}\right)
$$

dann sagen die Relationen (B) der Nr. 3 aus, dass für beliebige $\lambda$ und alle Indicespaare $p, q=1 \ldots n$ die Identität

$$
\begin{aligned}
0 & \equiv \sum_{I}^{n}\left(-L_{a}^{s p} L_{a^{\prime}}^{q s}+L_{a^{p}}^{s p} L_{a}^{q s}\right) \\
& +\sum_{I}^{v} L_{\mu+1}^{j p} H_{a a^{\prime}}^{q z}
\end{aligned}
$$

stattfindet. Hieraus und aus den Entwickelungen der Nr. 14 ergiebt sich, dass die folgenden beiden Matrices: 


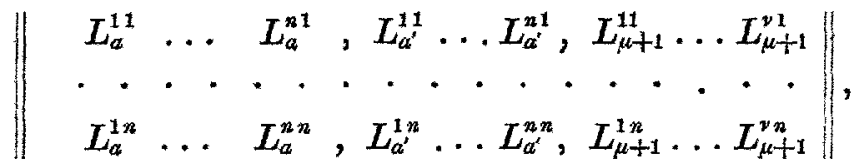

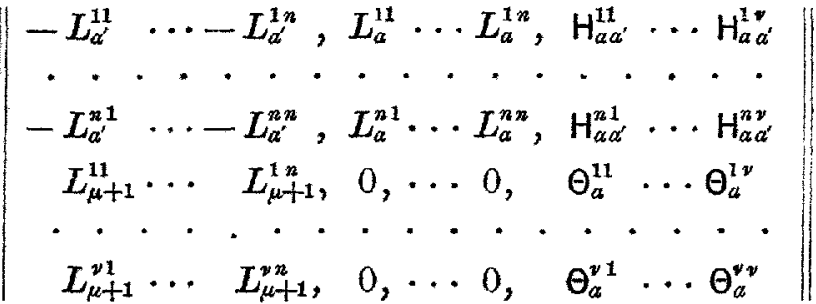

für beliebige $\lambda$ einander correspondiren, und zwar ist jede $n+\nu$ reihige Determinante von $(\delta)$ bis aufs Zeichen identisch gleich der $\mathrm{za}$ ihr complementären $n$-reihigen Determinante von $(\gamma)$ multiplicirt mit der Determinante

$$
\left|\theta_{a}^{i k}\right| * i, k=1,2 \ldots \nu
$$

18. Wir machen nun für den Augenblick die Annahme, dass wenigstens eine der $\mu$ Gleichungen

$$
\Delta_{a} \equiv\left|\begin{array}{ccc}
L_{a}^{11} & \cdots & L_{a}^{n 1} \\
\cdot & \cdot & \cdot \\
L_{a}^{1 n} & \cdots & L_{a}^{n n}
\end{array}\right|=0
$$

nicht für beliebige Werthe $\lambda_{\mu+1} \ldots \lambda_{m}$ mehrfach zählende Wurzeln $\lambda_{a}$ besitze; dass diese Voraussetaung ,im Allgemeinen" zutrifft, folgt schon daraus, dass sich aus den Bedingungen (B), (C) der Nr. 3 für die Coefficienten eines einzelnen Schemas $\Delta_{a}$ keine Relationen ableiten lassen.

Es genüge also die dem Index $a$ entsprechende Gleichung (7) der genannten Bedingung; substituiren wir nun in die Relation

$$
K_{\alpha}=0
$$

für $\lambda_{\mu+1} \ldots \lambda_{m}$ beliebige Constante $\lambda_{\mu+1}^{0} \ldots \lambda_{m}^{0}$ und bezeichnen wir mit $\lambda_{a b}^{0}$ irgend eine der $n-\nu$ Wurzeln der so erhaltenen algebraisehen Gleichung für $\lambda_{a}$, so dürfen wir nach dem eben gesagten annehmen, dass für die Werthe $\lambda_{a}^{0}, \lambda_{\mu+1}^{0} \ldots \lambda_{m}^{0}$ nicht alle ersten Hauptunterdeterminanten von $\Delta_{a}$ verschwinden; es sei etwa der zum Element $L_{a}^{11}$ gehörige Minor nicht null. Ist jetzt $\left(\alpha^{\prime}\right)$ die Matrix, die aus $(\alpha)$ entsteht, wenn man $\mathrm{ihr}$ die $n+1^{\text {te }}$ Colonne von $(\gamma)$ hinzufügt, so verschwinden alle $n$-reihigen Determinanten von $\left(\alpha^{\prime}\right)$, wenn man $\lambda_{a}, \lambda_{\mu+1} \ldots \lambda_{m}$ resp. durch $\lambda_{a}^{0}, \lambda_{\mu+1}^{0} \ldots \lambda_{m}^{0}$ und $\lambda_{\alpha^{\prime}}$ durch einen Werth $\lambda_{a}^{0}$ ersetzt, der sich durch $\lambda_{a}^{0} \ldots \lambda_{m}^{0}$ rational darstellen lässt. Nach dem 
Ergebniss der vor. Nummer verschwinden nun für die genannten Werthe der $\lambda$ auch alle $n+\nu$-reihigen Determinanten von $(\delta)$, welche zu den (auch in $(\gamma)$ enthaltenen) Determinanten von $\left(\alpha^{\prime}\right)$ bezw. complementär sind, d. h. die $n+2^{\text {te }}, n+3^{\text {te }} \ldots 2 n^{\text {to }}$ Colonne miteinander gemein haben, mithin, da unter den $n-1$-reihigen aus diesen Colonnen gebildeten Determinanten auch der oben genannte nicht verschwindende Minor von $\Delta_{a}$ sich vorfindet, überhaupt alle $n+\nu$-reihigen Determinanten von $(\delta)$. Aber die Determinante (6) ist für das wiederholt genannte Werthsystem der $\lambda$ von Null verschieden, da sonst wegen:

$$
\Delta_{\alpha} \equiv \mathcal{K}_{a} \cdot\left|\Theta_{a}^{i k}\right|
$$

die Gleichung (7) für $\lambda_{\mu+1}=\lambda_{\mu+1}^{0} \ldots \lambda_{m}=\lambda_{m}^{0}$ entgegen unserer Voraussetzung die mehrfach zählende Wurzel $\lambda_{a}^{0}$ besässe; es giebt also nach Nr. 17 zu jedem beliebigen System von Constanten $\lambda_{\mu+1}^{0} \ldots \lambda_{m}^{0} n-v$ verschiedene Werthepaare $\lambda_{a}^{0}, \lambda_{a}^{0}$ von der Beschaffenheit, dass diese Werthe der $\lambda$ alle n-reihigen Determinanten von $(\gamma)$ annulliren. Lässt man nun den Index $a^{\prime}$ alle Kahlen $1 \ldots \mu$ mit Ausnahme von $a$ durchlaufen, so folgt die Richtigkeit unseres Theorems unter den zu Anfang dieser Nr. gemachten Voraussetzungen.

18. Besitzen alle $\mu$ Gleichungen (7) für beliebige Werthe von $\lambda_{\mu+1} \ldots \lambda_{m}^{*}$ mehrfach zählende Wurzeln, so denken wir uns die Constanten $P_{a g}^{b h}$ unendlich wenig derart varïrt, dass diese Besonderheit nicht mehr stattfindet, die Bedingungen (B), (C) der Nr. 3 aber wach wie vor erfüllt sind. Für die charakteristische Matrix, die mit Hülfe der variirten Grössen $P_{a g}^{b k}$ gebildet wird, gilt nun das Theorem der Nr. 12; durch Rückübergang ergiebt sich sofort, dass die Dimensionsund Ordnungszahl der zur ursprünglichen Matrix gehörigen Punktmannigfaltigkeit $M$ nicht kleiner sind als bezw. $m-\mu-1$ und $n-\nu$, also nach Nr. 16 mit diesen Zahlen übereinstimmen, vorausgesetzt, dass eventuell irreducible $m-\mu-1$-fach ausgedehnte Bestandtheile von M mit geeigneter Vielfachheit in Rechnang gezogen werden.

19. Wir schreiben

$$
\Omega_{i j}^{k} \equiv \lambda_{1} \mathrm{P}_{i 1}^{k}+\lambda_{2} \mathrm{P}_{i 2}^{k}+\cdots+\lambda_{\mu} \mathrm{P}_{i \mu}^{k}+\lambda_{j} \mathrm{P}_{i j}^{k}
$$

und bilden die $m-\mu$ Matrices:

$j=\mu+1 \cdots m$.

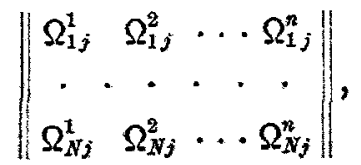

deren $x$-reihige Determinanten nach dem Theorem der $\mathrm{Nr}, 12$ resp. für $n-v$ im allgemeinen verschiedene Werthsysteme 


$$
\Lambda_{1 j}^{(x)}, \Lambda_{2 j}^{(x)} \cdots \Lambda_{\mu j}^{(x)} \quad(x=1,2 \cdots n-\nu)
$$

der Verhältnisse:

$$
-\lambda_{1}: \lambda_{j},-\lambda_{2}: \lambda_{j}, \cdots-\lambda_{\mu}: \lambda_{j}
$$

sämmtlich verschwinden. Es sei nun $(E)$ die aus $N$ Zeilen und $(m-\mu) n$ Colonnen bestehende Matrix, die durch Nebeneinanderstellnng der $m-\mu$ Schemata $\left(E_{\mu+1}\right) \ldots\left(\mathcal{E}_{m}\right)$ erhalten wird. Wir nehmen für den Augenblick an, dass alle n-reihigen Determinanten von $(E)$ verschainden, wenn in jedem der Theilschemata $\left(E_{j}\right)$ die Verhältnisse

$$
-\lambda_{1}: \lambda_{j} \cdots-\lambda_{\mu}: \lambda_{j}
$$

resp. durch die Grössen (6) ersetzt werden, vorausgesetat, dass der Index $(x)$ in allen Theilschematen gleich gewählt wird und der Reihe nach die Werthe 1, 2, ..n-v annimmt. Durch diese Voranssetzung legen wir den Grössen $P_{i j}^{k}$ natürlich eine Serie nener algebraischer Bedingungen auf. Sind diese erfüllt, so zerfält die Punktmannigfaltigkeit $M$ der Nr. 22 in $n-\nu$ lineare $m-\mu-1$-fach ausgedehnte Punktmannigfaltigkieiten, die bez. durch die Gleichungen:

$$
\lambda_{1}+\sum_{\mu+1}^{m} \Lambda_{1 j}^{(x)} \lambda_{j}=0 \cdots \lambda_{\mu}+\sum_{\mu+1}^{m} \Lambda_{\mu j}^{(x)} \lambda_{j}=0 ; \quad x=1 \cdots n-v
$$

definirt werden; in der That folgt aus anseren Voraussetzungen sofort, dass alle $n$-reihigen Determinanten von (D) für beliebige Werthe der Variabeln $\lambda_{\mu+1}, \lambda_{\mu+2} \ldots \lambda_{\mu}$ verschwinden, wenn $\operatorname{man}$ für $\lambda_{1}, \ldots \lambda_{\mu}$ ihre aus (7) hervorgehenden Werthe substitnirt.*)

Im Falle $\mu=1$ zerfallt also unter den gemachten Annahmen die charakteristische Form $K$ in $n-v$ lineare Factoren, d. h. man hat identisch:

$$
\begin{gathered}
K \equiv \pm \Lambda_{1} \Lambda_{2} \ldots \Lambda_{n-\gamma} \\
\Lambda_{x} \equiv \lambda_{1}+\Lambda_{2}^{(x)} \lambda_{2}+\Lambda_{s}^{(x)} \lambda_{2}+\cdots+\Lambda_{m}^{(x)} \lambda_{m}
\end{gathered}
$$

hierin bedeuten $\Lambda_{s}^{(1)} \ldots \Lambda_{s}^{(n-v)}$ die Werthe des Verhältnisses $-\lambda_{1}: \lambda_{s}$, welehe die Form $K$ annulliren, nachdem man darin alle $\lambda_{i}$ mit Ausnahme von $\lambda_{1}$ und $\lambda_{2}$ durch Null ersetzt bat.

\section{Capitel III.}

\section{Die Charakteristiken.}

20. Durch ein System von Gleichangen zwischen den Grössen $x, z, p_{i}^{z}$, welebes $x_{m}, z^{1} \ldots z^{n}, p_{1}^{1} \ldots p_{m}^{n}$ als Functionen der Variabeln $x_{1}, x_{2} \ldots x_{m-1}$ auszudrücken gestattet, sei eine beliebige Integralmannigfaltigkeit $\boldsymbol{M}_{m-1}$ des gegebenen Involutionssystems $\boldsymbol{J}$ definirt

*) Das Theorem lässt sich nicht umkehren. 
( $\nabla$ l. Nr. 10). Die genannten Functionen von $x_{1} \ldots x_{m-1}$ genügen dann den aus den Relationen

$$
d z^{\bar{k}}=p_{1}^{k} d x_{1}+\cdots+p_{m}^{i} d x_{m}
$$

hervorgehenden Differentialgleichungen:

$$
\frac{\partial z^{k}}{\partial x_{l}}-p_{m}^{k} \frac{\partial x_{m}}{\partial x_{l}}-p_{l}^{k}=0 ; \quad k=1 \ldots n ; l=1, \ldots m-1 .
$$

Da nach $\mathrm{Nr} .10$ durch eine beliebige Integral- $M_{m-1}$ von $J$ im Allgemeinen eine und nur eine Integral- $M_{m}$ hindurehgeht*), so ist durch jedes Element $x, z, p_{i}^{k}$ unserer $\boldsymbol{M}_{m-1}$ ein einziges System von Werthen $r_{i j}^{k}$ definirt, welche den Gleichungen

$$
f_{i l}=0 \text { (vgl. Nr.6) }
$$

sowie den aus den Beziehungen

$$
d p_{i}^{k}=r_{i 1}^{k} d x_{1}+\cdots+r_{i m}^{k} d x_{m}
$$

folgenden Relationen:

$$
\begin{gathered}
\frac{\partial p_{l}^{k}}{\partial x_{j}}-r_{l m}^{k} \frac{\partial x_{m}}{\partial x_{j}}-r_{b j}^{k}=0 ; \\
j=1 \ldots m-1 ; l=1 \ldots m ; k=1 \ldots n,
\end{gathered}
$$

Genūge leisten. Durch Elimination der $\eta_{i j}^{\pi_{j}^{k}}$ folgen hieraus, beilänfig bemerkt, die für jede Element- $M_{m-1}$ gültigen Beziehungen:

$$
\begin{gathered}
\frac{\partial p_{l}^{k}}{\partial x_{j}}-\frac{\partial p_{j}^{k}}{\partial x_{2}}+\frac{\partial p_{m}^{k}}{\partial x_{j}} \frac{\partial x_{m}}{\partial x_{i}}-\frac{\partial p_{m n}^{k}}{\partial x_{2}} \frac{\partial x_{m}}{\partial x_{j}}=0 \\
(l, j=1,2, \ldots m-1 ; k=1 \ldots n)
\end{gathered}
$$

die sich übrigens auch leicht aus (2) ableiten lassen.

21. Aus den Combinationen

$$
f_{i l}+\frac{\partial x_{m}}{\partial x_{l}} f_{i m}=0 \quad(l=1 \ldots m-1, i=1 \ldots n)
$$

fallen die Grössen $r_{i j}^{k}$ vermöge (5) herans, und es folgen die Beziehungen

$$
M_{i l}+\frac{\partial x_{m}}{\partial x_{i}} M_{i m}+\sum_{1}^{n} \sum_{I}^{m} P_{i s}^{k} \frac{\partial p_{s}^{k}}{\partial x_{l}}=0
$$

die natürlich für jede Integral- $\boldsymbol{M}_{m-1}$ von $\mathcal{J}$ identisch erfüllt sind. Um die $r_{i j}^{k}$ aus (3) und (5) zu berechnen, genügt es sonach, die Relationen

$$
f_{1 m}=0, f_{2 m}=0 \ldots f_{N m}=0
$$

zu betrachten, aus denen vermöge (5) für die Ableitungen $m_{m}^{k}$ das folgende Gleichungssystem hervorgeht:

*) Wobei allerdings voransgesetzt wird, dass die Relation, die $x_{m}$ als Function von $x_{1} \ldots x_{m-1}$ darstellt, nach $x_{1}$ auflöstar sei. 
Hierin ist

$$
\begin{gathered}
M_{i m}+\sum_{1}^{n} \sum_{1}^{m-1} P_{i s}^{k} \frac{\partial p_{m}^{k}}{\partial x_{s}}-\sum_{1}^{n} \Pi_{i}^{k} r_{m m}^{k}=0 \\
(i=1,2 \ldots N) .
\end{gathered}
$$

$$
\Pi_{i}^{k} \equiv-\mathrm{P}_{i m}^{k}+\sum_{I}^{m-1} \mathrm{P}_{i j}^{k} \frac{\partial x_{m}}{\partial x_{j}}
$$

gesetzt. Nach dem oben gesagten lassen sich die Unbekannten $r_{m m}^{k}$ hieraus eindeutig bestimmen, worauf die übrigen Ableitungen $r_{i j}^{k}$ mit Hülfe von (5) ermittelt werden.

Nennen wir ein Wertbsystem $x, z, p_{r}^{s}, r_{i j}^{z}$ ein ,Element 2. Ordnung", welches das Element 1. Ordnung $x, z, p_{r}^{*}$,enthält", so können wir sagen: $z u$ jedem Element 1. 0 . der betrachteten Integral- $M_{m-1}$ gehört ein und nur ein Element 2. 0., das jenes Element 1. 0. und alle $\infty^{m-8}$ dazu benachbarten Elemente von $\bar{M}_{m-1}$ enthält, und den Relationen (3) Genüge leistet.

Es existiren im Allgemeinen zu jedem Werthsystem $x, z, p_{i}^{\bar{k}}$ unserer Integral- $M_{m-1} \mathrm{~N}-n$ linear unabhängige Lösungssysteme

$$
l_{1, s}, l_{2, s} \ldots l_{N, s} \quad(s=1 \ldots N-n)
$$

der linearen Gleichungen in den Unbekannten $l_{i}$ :

$$
\sum l_{i} \Pi_{i}^{k}=0 \quad(k=1 \ldots n)
$$

und es ergiebt sich somit, dass jede beliebige Integral- $\boldsymbol{M}_{m-1}$ der betrachteten Art auch die aus (8) folgenden $\mathrm{N}-n$ Relationen

erfüllen muss.

$$
\sum_{1}^{N}\left(M_{i m}+\sum_{1}^{n} \sum_{1}^{m-1} P_{i j}^{k} \frac{\partial p_{m}^{k}}{\partial x_{j}}\right) l_{i, s}=0
$$

22. Anders verhält sich die Sache, wenn für alle Elemente $x, z, p_{i}^{k}$ der betrachteten Integral- $M_{m-1}$ die sämmtlichen $n$-reihigen Determinanten der aus den Grössen $\Pi_{i}^{k}$ gebildeten Matrix verschwinden, was wir durch die symbolische Gleichung:

$$
\left\|\Pi_{i}^{k}\right\|=0
$$

andenten wollen. Die Relationen (3) und (5) reichen jetzt zur Bestimmung der Unbekannten $\dot{r}_{i j}^{k}$ nicht mehr hin. Sollen die Gleichnngen (8) überhaupt von endlichen Werthsystemen der $r_{i j}^{k}$ befriedigt werden, so müssen auch alle Relationen (10), deren Anzahl nunmehr $>\mathrm{N}-n$ ist, für die Elemente unserer $\boldsymbol{M}_{m-1}$ erfüllt sein. Eine derartige $\boldsymbol{M}_{m-1}$. heisse eine $m-1$ fach ausgedehnte charakteristische Mannigfaltigkeit oder Charakteristik des Involutionssystems $J$ und werde generell mit $C_{m-1}$ bezeichnet. 
Eine $C_{m-1}$ ist demnach dadurch gehennzeichnet, dass es unbegrenzt viele Elemente 2. Ordnung giebt, welche ein beliebiges Element 1. 0 . von $C_{m-1}$, sovie alle dazu benachbarten enthalten und den Beziehungen (3) genügen.

23. Es erhebt sich vor allem die Frage, ob Charakteristiken $C_{m-\text { : }}$ für ein beliebig gegebenes Involutionssystem $J$ überhaupt existiren. Wir machen zunächst die Annahme $\mu=1$. Nach den Ergebnissen der Nr. 15 sind dann die Bedingungen (11) äquivalent mit der einen Gleichnng:

$$
K\left(\frac{\partial x_{m}}{\partial x_{1}}, \frac{\partial x_{m}}{\partial x_{2}}, \cdots \frac{\partial x_{m}}{\partial x_{m-1}},-1\right)=0
$$

Drüeken wir hierin mit Hülfe der Definitionsgleichungen einer beliebigen Integral- $\boldsymbol{M}_{m}$ von $J$ die Grössen $z, p_{i}^{k}$ als Functionen von $x_{1} \ldots x_{m}$ aus, so ergiebt sich eine partielle Differentialgleichung I. 0 . mit der unbekannten Function $x_{m}$ and der Independenten $x_{1} \ldots x_{m-1}$. Sind die $x_{i}$ cartesische Punktcoordinaten eines $R_{m}$, so sind dem Grade $n-\nu$ der Gleichung (12) entsprechend nach bekannten Sätzen durch eine im $R_{m}$ beliebig gewählte Punktmannigfaltigkeit $x$ von $m-2$ Dimensionen $n-v$ sie enthaltende Zweige

$$
x_{m}=\psi_{i}\left(x_{1} \ldots x_{m-1}\right) \quad i=1 \ldots n-v
$$

einer $m$ - 1-dimensionalen Integralmannigfaltigkeit von (12) festgelegt; dieselbe projicirt sich auf die oben betrachtete Integral- $\boldsymbol{M}_{m}$ von $\boldsymbol{J}$ offenbar als eine Charakteristik, d. h. jede der Relationen (13) stellt zusammen mit den Definitionsgleichungen von $M_{m z}$, die $z, p_{i}^{k}$ als Functionen der $x$ bestimmen, eine $C_{m-1}$ von $J$ dar. Da sich ebenso die Mannigfaltigkeit $\pi$ als beliebige $m-2$-dimensionale Mannigfaltigkeit auf $\boldsymbol{M}_{m}$ projicirt, so haben wir das Theorem:

„Im Falle $\mu=1$ gehen durch eine beliebige $m-2$-fach ausgedehnte Elementenmannigfaltigkeit, die auf einer Integral- $M_{m}$ des Involutionssystems $\boldsymbol{J}$ verläuft, im allgemeinen $n-\nu$ vollständig bestimmte Zweige einer ganz auf $\boldsymbol{M}_{m}$ enthaltenen Charakteristit $C_{m-1}$ hindurch."

24. Ist $\mu>1$, so sind die Relationen (11) mit $\mu$ versehiedenen partiellen Differentialgleichungen für $x_{m}$ äquivalent. Auf die Frage, ob diese Gleichungen gemeinsame Integrale besitzen, gehen wir an dieser Stelle nicht näher ein.

25. Fs ist leicht, die Entwickelungen der Nrn. 22 and 23 zu verallgemeinern. Betrachten wir zunächst, falls $m>2$, eine Integral$M_{m \rightarrow 2}$ von $J$, die auf irgend einer Integral- $M_{m}$ verlaufen möge, and durch deren Definitionsgleichungen die Grössen $x_{m-1}, x_{m}, z, p$ als Functionen der Variabeln $x_{1}, x_{2}, \ldots x_{m-2}$ dargestellt seien, Aus den Relationen (3) und 


$$
\frac{\partial p_{l}^{k}}{\partial x_{j}}-r_{l, m-1}^{k} \frac{\partial x_{m-1}}{\partial x_{j}}-r_{b, m}^{k} \frac{\partial x_{m}}{\partial x_{j}}-r_{l, j}^{k}=0
$$

ergeben sich sofort die folgenden:

$$
\begin{gathered}
M_{i l}+\frac{\partial x_{m-1}}{\partial x_{l}} M_{i, m-1}+\frac{\partial x_{m}}{\partial x_{l}} M_{i m}+\sum_{1}^{n} \sum_{l}^{m} P_{i s}^{x} \frac{\partial p_{s}^{k}}{\partial x_{l}}=0 \\
(l=1,2 \ldots m-2)
\end{gathered}
$$

welche für ein Integral- $\boldsymbol{M}_{m-2}$ eo ipso erfüllt sind, ferner noch die Beziehungen :

$$
\begin{gathered}
M_{i, m-1}+\sum_{1}^{n} \sum_{1}^{m-2} \mathrm{P}_{i s}^{k} \frac{\partial p_{m-1}^{k}}{\partial x_{s}}=\sum_{1}^{n} \Phi_{i}^{k} r_{m-1, m-1}^{k}+\sum_{1}^{k} \psi_{i}^{k} r_{m-1, m}^{k}, \\
M_{i, m}+\sum_{1}^{k} \sum_{1}^{m-2} \mathrm{P}_{i s}^{k} \frac{\partial p_{m}^{k}}{\partial x_{s}}=\sum_{1}^{n} \Phi_{i}^{k} r_{m-1, m}^{k}+\sum_{1}^{k} \psi_{i}^{k} r_{m m}^{k}
\end{gathered}
$$

worin

$$
\begin{aligned}
& \Phi_{i}^{k} \equiv-\mathrm{P}_{i, m-1}^{k}+\sum_{1}^{m-2} \mathrm{P}_{i s}^{k} \frac{\partial x_{m-1}}{\partial x_{s}}, \\
& \Psi_{i}^{k} \equiv-\mathrm{P}_{i m}^{k}+\sum_{1}^{m-2} \mathrm{P}_{i s}^{k} \frac{\partial x_{m}}{\partial x_{s}}
\end{aligned}
$$

gesetzt wird. Wir wollen die betrachtete Integral- $M_{m-2}$ eine $C_{s m-2}$, d. h. eine,$m-2$-fach ausgedehnte Charakteristik" nennen, wenn alle $n$-reihigen Determinanten der Matrix

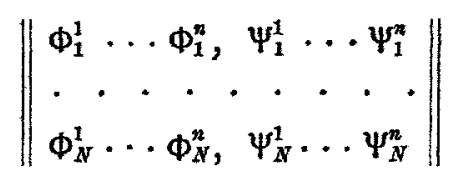

identiseh Null sind. Diese Annahme liefert ein System partieller Differentialgleichungen I. 0. mit den abhängigen Variabeln $x_{m-1}, x_{m}$ und den unabhängigen Variabeln $x_{1} \cdots x_{m-2}$, wenn man die in den Coefficienten $\mathrm{P}_{i z}^{k}$ auftretenden Grössen $z, p$ durch die Ausdrücke in $x_{1} \ldots x_{m}$ ersetzt, welehe der oben erwähnten Integral- $M_{m}$ zukommen. Die erbaltenen partiellen Differentialgleichungen sind aber selbst im Falle $\mu=1$ (und a fortiori für $\mu>1$ ) im Allgemeinen nicht miteinander vertrïglich. Dies lehrt schon der einfache Fall $\mu=1, \nu=0, m=3$; die charakteristische Form $K$ ist hier identisch mit der $n$-reihigen Determinante, die ans dem (nunmehr quadratischen) Schema (D) p. 554 gebildet wird; d. h. mit einer vollständig allgemeinen Form $n$. Grades in $\lambda_{1} \lambda_{2} \lambda_{3}$, während das Verschwinden aller $n$-reihigen Determinanten der zugehörigen Matrix (17) uach Nr. 19 die Zerlegbarkeit der Form $K$ im Gefolge bätte. 
Auch für Involutionssysteme $\mu=1$ enthält somit eine beliebige Integral- $M_{m}$ im allgemeinen keine Charakteristiken $C_{m-2}$.

26. In der vorliegenden ersten Abhandlung werden fortan nur solche Involutionssysteme $J$ betrachtet, für welche die Zahl $\mu$ den Werth eins besitzt. Nach Nr. 11 kann jedes beliebige Involutionssystem mit Hülfe der Mayer'schen Transformation auf diesen Fall zurückgeführt werden.

27. Durch leichte Verallgemeinerung der in den vor. Nummern durchgefübrten Rechnungen gelangt man zu einer ganz analogen Definition der Charakteristiken $C_{m-3}, C_{m-4}$ etc.; wir betrachten ausführlich den Fall der Charakteristiken $C_{1}$.

Es seien die Grössen $x_{2}, x_{3} \ldots x_{m}, z, p$ als Functionen der unabhängigen Variabeln $x_{1}$ so bestimmt, dass die Relationen

$$
d z^{k}=p_{1}^{k} d x_{1}+\cdots+p_{m}^{k} d x_{m} \quad(k=1 \ldots n)
$$

identisch bestehen. Die so definirte Element- $M_{1}$, die wir gelegentlich auch als "Streifen ${ }^{\prime \prime}$ bezeichnen wollen, ist eine Integralmannigfaltigkeit von $J$, wenn die Bedingungen

$$
d f_{1}=0, d f_{2}=0 \ldots d f_{n+v}=0
$$

erfüllt sind, und ausserdem ein Element des Streifens die Relationen (A) erfüllt. Ist $\boldsymbol{M}_{m}$ eine $m$-fach ausgedehnte Integralmannigfaltigkeit, welche unsere $M_{1}$ enthält, so ist jedem Element von $M_{1}$ durch die $\boldsymbol{M}_{m}$ ein bestimmtes System von Werthen $r_{i j}^{k}$ zugeordnet, welche den Relationen (3) sowie den folgenden:

$$
d p_{i}^{\bar{k}}=r_{i 1}^{k} d x_{1}+\cdots+r_{i m}^{k} d x_{m} \quad(k=1 \cdots n, i=1 \cdots m)
$$

Genüge leisten. Die Elimination der Grössen $r_{i 1}^{k}$ ans (3) und (20) führt auf die schon betrachteten Beziehungen (19), sowie auf die nachfolgenden $m-1$ Systeme von je $\mathrm{N}=n+v$ Gleichungen:

$\left.\left(F_{h}\right)\right\}$
$h=2,3 \cdots a b$$\left\{\begin{array}{c}\mathrm{M}_{i h}+\sum_{1}^{k} \mathrm{P}_{i 1}^{k} \frac{d p_{h}^{k}}{d x_{1}}+\sum_{1}^{n} \sum_{2}^{m} r_{h j}^{k}\left(\mathrm{P}_{i j}^{k}-\frac{d x_{j}}{d x_{1}} \mathrm{P}_{i 1}^{\bar{k}}\right)=0 \\ (i=1,2 \ldots \mathrm{N}) .\end{array}\right.$

Wir nennen den Streifen $M_{1}$ einen "charalteristischen Streifen" oder eine $C_{1}$ von $J$, wenn sich die $N$ Gleichungen eines jeden der Systeme $\left(F_{2}\right) \ldots\left(F_{m}\right)$ auf nur $n-1$ in Bezug auf die Unbekannten $r_{i j}^{k}$ linear unabhängige Relationen reduciren.

28. Nach Nr, 19 hat man für eine $C_{1}$ die Beziehungen

$$
\frac{d x_{2}}{d x_{1}}=\Lambda_{2}^{(x)}, \frac{d x_{3}}{d x_{1}}=\Lambda_{3}^{(x)} \cdots \frac{d x_{m}}{d x_{1}}=\Lambda_{m}^{(x)},
$$

unter $*$ einen der Indices $1,2, \ldots n-\nu$ verstanden, und es müssen die in jener Nr. angegebenen algebraischen Bedingungsgleichungen 
erfüllt sein, d. h. es müssen alle $n$-reihigen Determinanten der aus $n+v$ Zeilen und $n(n-1)$ Colonnen bestehenden Matrix

$\left(G_{x}\right)$

$$
\left\|\begin{array}{c}
P_{12}^{1}-\Lambda_{2}^{(x)} P_{11}^{1}, \ldots P_{12}^{n}-\Lambda_{2}^{(x)} P_{11}^{n} \ldots P_{1 m}^{n}-\Lambda_{m}^{(x)} P_{11}^{n} \\
\cdot \cdot \cdot \cdot \cdot \cdot \cdot \cdot \cdot \cdot \cdot \cdot \cdot \cdot \cdot \cdot \cdot \cdot \cdot \\
P_{N 2}^{1}-\Lambda_{2}^{(x)} P_{N 1}^{1}, \ldots P_{N 2}^{n}-\Lambda_{2}^{(x)} P_{N 1}^{n} \ldots P_{N m}^{n}-\Lambda_{m}^{(x)} P_{N 1}^{n}
\end{array}\right\|
$$

für alle Indices $x=1,2 \ldots n-v$ vermöge (A) Null sein.

Die charakteristische Form $K$ zerfällt dann in $n-1$ Linearfactoren $\Lambda_{1} \ldots \Lambda_{n-v}$, die wir fortab ausdriicklich als von einander verschieden voraussetzen wollen. Dann ist klar, dass in keinem der $n-\nu$ Schemata $\left(G_{x}\right)$ alle $n-1$-reihigen Determinanten verschwinden können, da andernfalls der Factor $\Lambda_{x}$ in $K$ mehrfach auftreten würde.

29. Unter den soeben gemachten Annahmen besitzen die $n(m-1)$ Gleichungen in den Unbekannten $l_{1}^{(x)} \ldots l_{N}^{(x)}$

$$
\sum_{1}^{N} l_{i}^{(x)}\left(\mathrm{P}_{i j}^{k}-\Lambda_{j}^{(x)} \mathrm{P}_{i 1}^{k}\right)=0, j=2 \ldots m, k=1 \ldots n
$$

für jeden der Indices $x=1,2, \ldots, n-v$ genau $v+1$ linear unabhängige Lösungssysteme

$$
l_{s s}^{(x)} l_{2 s}^{(x)} \ldots l_{N s}^{(x)} \quad(s=1,2, \ldots v+1) .
$$

Die Elimination der Unbekannten $r_{i j}^{k}$ aus den Relationen $\left(F_{h}\right)$ führt sonach auf die folgenden totalen Differentialgleichungen, denen jeder Streifen $C_{1}$ ebenfalls genügen muss:

$$
\begin{aligned}
& \sum_{1}^{N} l_{i s}^{(x)} M_{i k}+\sum_{1}^{N} \sum_{1}^{n} l_{i s}^{(x)} \mathrm{P}_{i 1}^{k} \frac{d p_{h}^{k}}{d x_{1}}=0, \\
& (h=2, \ldots m ; s=1,2, \ldots, \nu+1) .
\end{aligned}
$$

Aber diese $(\nu+1)(m-1)$ Relationen sind nicht alle unabhängig von den Gleichungen (19); denn unter den Lösungen $l_{i}^{(*)}$ der Gleichungen (22) befinden sich, wie aus den Bemerkungen der $\mathrm{Nr} .15$ sofort folgt, $u$. a. die sämmtlichen $(m-1) \nu$ Grössensysteme:

$$
\begin{gathered}
\alpha_{1 j}^{s}-\Lambda_{j}^{(x)} \alpha_{11}^{s}, \quad \alpha_{2 j}^{s}-\Lambda_{j}^{(x)} \alpha_{21}^{s} \cdots \alpha_{N j}^{s}-\Lambda_{j}^{(x)} \alpha_{N_{1}}^{s}, \\
(s=1 \ldots \nu ; j=2 \ldots m)
\end{gathered}
$$

mithin sind die $\nu$ Ausdrücke

$$
\sum_{z}^{m} \sum_{i}^{N}\left(\alpha_{i j}^{*}-\Lambda_{j}^{(x)} \alpha_{i 1}^{*}\right) M_{i j}+\sum_{2}^{m} \sum_{1}^{N} \sum_{1}^{n} k \mathrm{P}_{i 1}^{k}\left(\alpha_{i j}^{*}-\Lambda_{j}^{(x)} \alpha_{i 1}^{s}\right) \frac{d p_{j}^{k}}{d x_{i}}
$$

lineare Combinationen der linken Seiten von (23). Aber diese Aus- 
drücke sind andererseits zufolge der Relationen (4) der Nr. 15 mit den folgẻnden identisch:

$$
-\sum_{1}^{N} i \alpha_{i 1}^{s} \frac{d f_{i}}{d x_{1}}
$$

dabei ist in $d f_{i}$ für $d z^{k}$ sein ans (18), (21) folgender Werth

$$
\left(p_{1}^{k}+p_{2}^{k} \Lambda_{2}^{(x)}+\cdots+p_{m}^{k} \Lambda_{m}^{(x)}\right) d x_{1}
$$

einzusetzen. Demnach sind $\nu$ unabhängige Linearcombinationen der Gleichungen (23), - und wie leicht ersichtlich anch nicht mehr eine Folge von (19). Also sind die Gleichungen (19), (23) für jeden Index $x$ zusammen nur mit $(m-1)(v+1)+n$ unabhängigen Relationen für die Ableitungen $\frac{d p_{j}^{k}}{d x_{1}}$ äquivalent.

Wir bezeichnen ein Involutionssystem der Form (A) als ein Normal. system, wenn die Zahl $\mu=1$ ist und die algebraischen Bedingungsgleichungen der Nr.19 erfüllt sind. Dann können wir folgenden Satz aussprechen:

„Jedes Normalsystem besitzt $n-\nu$ im allgemeinen verschiedene Systeme charakteristischer Streifen $C_{1}$ ".

30. Wir wollen die $n-\nu$ Systeme von Definitionsgleichnngen der $C_{1}$ der Uebersicht halber noch einmal zusammenstellen:

$\left(\mathrm{H}_{*}\right)$

$$
x=1,2, \quad\left\{\begin{array}{l}
\text { a) } \frac{d x_{2}}{d x_{1}}=\Lambda_{2}^{(x)}, \ldots, \frac{d x_{m h}}{d x_{1}}=\Lambda_{m}^{(x)}, \\
\text { b) } \frac{d z^{k}}{d x_{i}}=p_{1}^{k}+\sum_{z}^{m} \Lambda_{j}^{(x)} p_{j}^{k}, \quad(k=1 \ldots n) \\
\text { c) } d f_{1}=0, d f_{2}=0, \ldots, d f_{x+\nu}=0, \\
\sum_{1}^{N} l_{i s}^{(x)} M_{i k}+\sum_{i}^{N} \sum_{1}^{n} l_{i s}^{(x)} P_{i 1}^{k} \frac{d p_{h}^{k}}{d x_{i}}=0, \\
(h=2,3, \ldots, m ; s=1,2, \ldots, \nu+1) .
\end{array}\right.
$$

Man kann die Gleichungen c) ans diesem System weglassen und die Grössen $p_{1}^{1} \ldots p_{1}{ }^{n}, p_{2}{ }^{1} \ldots p_{2}{ }^{p}$ nebst ihren Differentialen vermöge des aufgelösten Systems $\left(A^{\prime}\right)$ aus $\left(H_{x}\right)$ entfernen. Das so modificirte Gleichungssystem $\left(H_{x}\right)$ werden wir später mit $\left(H_{x}^{\prime}\right)$ bezeichnen. Es besteht aus $(m-1)(\nu+1)-\nu+n+m-1$ unabhängigen Gleichungen zwischen den $(m-1)+n+(m n-n-v)$ Ableitungen $\frac{d x_{j}}{d x_{1}}, \frac{d x^{k}}{d x_{1}}, \frac{d p_{g}^{h}}{d x_{i}} ;$ die Zahl der letateren ist somit um $(m-1)(n-\nu-1)$ grösser als die der ersteren, also gleich derselben nur im Falle $v=n-1$. 
„Ein Normalsystem $\nu=n-1$ besitzt demnach eine Schaar von eindimensionalen Charakteristiken, die nur von einer endlichen Parañeterzahl abhängt."

31. Ihrer Entstehungsweise nach sind die Definitionsgleichungen $\left(\mathrm{H}_{x}\right)$ der $C_{1}$ völlig äquivalent mit den folgenden:

$$
\begin{aligned}
& d x_{j}=\Lambda_{j}^{(x)} d x_{1}, \quad j=2 \ldots m \\
& d z^{k}=p_{1}^{k}+\sum_{2}^{m} p_{l}^{k} \Lambda_{l}^{(x)}, \quad k=1 \ldots n \\
& a p_{i}^{k}=r_{i 1}^{k}+\sum_{2}^{m} r_{i l}^{k} \Lambda_{l}^{(x)}, \quad k=1 \ldots n, \quad i=1 \ldots m
\end{aligned}
$$

wenn unter den $r_{i l}^{k}$ beliebige Grössen verstanden werden, die den Beziehungen (3) genügen. Jede eindimensionale Mannigfaltigkeit von „Pankten" $x_{1} \ldots x_{m}, z_{1} \ldots z_{n}$, welche den Differentialgleichungen (24) genügt und einer Integral $-M_{m}$ von $J$ angehört, bestimmt also mit den ihr zugehörigen Elementen 1. O. von $\boldsymbol{M}_{m}$ eine Charakteristik $C_{1}$; wir schliessen daraus sofort:

,Jede Integral- $\boldsymbol{M}_{m}$ des Normalsystems $J$ ist erzeugt von je $\infty^{m-1}$ Streifen $C_{1}$ eines jeden der $n-\nu$ Charakteristifensysteme, in dem Sinne, dass fü $x=1,2 \ldots, n-v$ durch jedes Element $x, z, p$ von $M_{m}$ ein und nur ein Streifen hindurchgeht, welcher dem $\varkappa^{\text {ten }}$ Charakteristikensystem angehört und ganz auf $\boldsymbol{M}_{m}$ enthalten ist."

32. Die partielle Differentialgleichung (12) der Charakteristiken $C_{m-1}$ unseres Normalsystems $J$ zerfällt in die $n-\nu$ linearen Gleichungen:

$$
\begin{gathered}
\frac{\partial x_{m}}{\partial x_{1}}+\Lambda_{2}^{(x)} \frac{\partial x_{m}}{\partial x_{2}}+\cdots+\Lambda_{m-1}^{(x)} \frac{\partial x_{m}}{\partial x_{m-1}}=\Lambda_{m}^{(x)}, \\
(x=1,2 \ldots n-\nu) .
\end{gathered}
$$

Ersetat man in den Functionen $\Lambda_{j}^{(x)}$ die Grössen $z, p$ vermöge der Definitionsgleichungen irgend einer Integral $-\boldsymbol{M}_{m}$ von $J$ durch Functionen der $x$, so entstehen lineare partielle Differentialgleichungen für $x_{m}$, deren "Charakteristiken" im gewöhnlichen Sinn durch (24) definirt werden. Man schliesst darans unmittelbar:

„Ein Normalsysten besitat $n-v$ verschiedene $C_{m-1}$-Systeme; die allgemeinste $C_{m-1}$, die auf einer beliebigen Integral- $M_{m}$ von $J$ enthalten ist, wird von $\infty^{m-2}$ Charalteristiken $C_{1}{ }^{(*)}$ erzengt, die ganz auf $\boldsymbol{M}_{m}$ verlaufen und bezw. von den Elementen einer beliebigen, auf $\boldsymbol{M}_{m}$ gelegenen $\boldsymbol{M}_{m-2}$ ausgehen."

Für die $m-s$-dimensionalen Charakteristiken $C_{m-s}$ hat man ebenso die $n-\nu$ Systeme von je $s$ Gleichungen: 


$$
\begin{gathered}
\frac{\partial x_{m-s+j}}{\partial x_{s}}+\Lambda_{2}^{(x)} \frac{\partial x_{m-s+j}}{\partial x_{2}}+\cdots+\Lambda_{m-s}^{(x)} \frac{\partial x_{m-s+j}}{\partial x_{m-s}}=\Lambda_{m-s+j}^{(x)}, \\
(j=1,2, s)
\end{gathered}
$$

woraus wie oben folgt:

"Es gibt für ein Normalsystem n-v verschiedene Systeme von Charahteristiken $C_{m-s}$; die allgemeinste, auf einer beliebigen Integral$M_{m}$ von $J$ verlaufende $C_{m-s}$ vird von $\infty^{m-s-1}$ Charaliteristiken $C_{1}^{(x)}$ erzeugt, aie bezw. von den Elementen einer willkürlichen, auf $\boldsymbol{M}_{m}$ gelegenen $M_{m-5-1}$ auslaufen."

\section{Capitel IV.}

\section{Reduction der Anzahl der Independenten.}

33. Aus den Entwickelungen der Nr. 30 und 31 ergibt sich für Normalsysteme, deren Zahl $v$ den Werth $n-1$ hat, eine Theorie, die derjenigen einer partiellen Differentialgleichung 1.0 . mit einer unbekannten Function vollkommen analog ist, und auch thatsächlich in dieselbe übergeht, wenn wir im vorigen Capitel die Annahme $n=1$, $\mu=1, \nu=0$ machen. Für $\nu=n-1$ gibt es nämljch einerseits nach Nr. 30 nur ein einziges System von eindimensionalen Charakteristiken, die durch gewöhnliche Differentialgleichungen definirt werden, und zwar ist jedes Flächenelement der durch (A) definirten Schaar auf eirer und nor einer $C_{1}$ enthalten; andererseits sind nach Nr. 31 auf jeder Integral- $\boldsymbol{M}_{m}$ je $\infty^{m-1} C_{1}$ gelegen, und es folgt:

Die allgemeinste Integral- $\boldsymbol{M}_{m}$ eines Normalsystems $\nu=n-1$ wird erzeugt von $\infty^{m-1}$ Charakteristiken $C_{1}$, die bezw. durch die $\infty^{m-1}$ Elemente einer beliebigen Integral- $\boldsymbol{M}_{n-1}$ bestimmt werden. Die Integration des Systems (A) ist so zurückgeführt auf diejenige eines gewöhnlichen Differentialgleichungssystems, auf ausführbare Operationen und auf die Herstellung der allgemeinsten Integral- $M_{n_{n-1}}$ von $J$, die nach Nr. 10 die Integration eines Hillfssystems von $n-1$ Gleichungen I. 0 . in $m-1$ Independenten und $n-1$ unbekannten Functionen erfordert.

34. Betrachten wir nunmehr den Fall $y<n-1$. Die um eins verminderte Zahl der Differentiale $d x_{1} . d x_{m}, d z^{1} \cdot d z^{n}, d p_{2}^{x+1} \ldots d p_{2}^{n}$, $d p_{3}^{1} \ldots d p_{m}^{n}$ übertrifft nunmehr nach Nr. 30 diejenige der unabhängigen Gleichungen in dem System $\left(H_{x}^{\prime}\right)$, and es sind gewisse Integrabilitätsbedingungen erforderlich, wenn sich aus den linken Seiten der (in den Differentialen homogen geschriebenen) Gleichnngen $\left(\mathrm{H}_{x}\right)$ lineare Combinationen der Form $d \psi^{(x)}$ sollen bilden lassen, unter $*$ irgend eine der Zahlen 1.n-v, unter $\psi^{(x)}$ eine Funetion der Grössen $x, z, p_{g}^{n}$ verstanden. Nehmen wir nun an, es sei eine solche Combination vorbanden, von der Beschaffenheit, dass sich die Gleichung

$$
\psi^{(x)}=0
$$


in der Form

auflösen lässt.

$$
-p_{2}^{\nu+1}+\varphi_{2}^{\nu+1}\left(x, z, p_{2}^{\nu+2} \ldots p_{2}^{n}, p_{3}^{1} \cdot p_{m}^{n}\right)=0
$$

Dasn bilden die $n+v+1$ Relationen:

$$
-p_{1 i}^{b}+\varphi_{1}^{b}=0,-p_{2}^{c}+\varphi_{2}^{c}=0(b=1 \ldots n ; c=1 \ldots \nu+1)
$$

wieder ein Involutionssystem, wenn man sich die Grösse $p_{2}^{v+1}$ aus den ersten $N$ Gleichungen vermöge der letzten eliminirt denkt. Denn der Umstand, dass $d \psi^{(x)}$ eine integrable Combination der linken Seiten des Systems $\left(H_{x}^{\prime}\right)$ ist, lässt sich nach $\mathrm{Nr} .31$ so ausdrücken: Der totale Differentialausdruck $d \psi^{(x)}$ verschwindet vermöge der Relationen (3), pag. 6, wenn man $d x_{i}, d z^{k}, d p_{g}^{h}$ durch ihre Ausdrücke (24), (25), (26) des vor, Cap. ersetzt. Schreiben wir demnach:

$$
\psi_{l}^{(x)} \equiv \frac{\partial \psi^{(x)}}{\partial x_{l}}+\sum_{1}^{n} \frac{\partial \psi^{(x)}}{\partial z^{k}} p_{l}^{k}+\sum_{g, h} \frac{\partial \psi^{(x)}}{\partial p_{g}^{h}} r_{g l}^{h},
$$

so besteht vermöge (A) eine Identität der Form

$$
\Psi_{1}^{(x)}+\Lambda_{2}^{(x)} \Psi_{2}^{(x)}+\cdots+\Lambda_{m}^{(\alpha)} \Psi_{m}^{(x)} \equiv \sum_{1}^{m} \sum_{1}^{N} \sigma_{i l} f_{i l}
$$

für alle Werthe der $r_{i j}^{k}$; also gibt es nach Nr. 6 zwischen den linken Seiten der $m(\mathbf{N}+1)$ Relationen, die durch partielle Ableitung aus:

$$
f_{1}=0 \ldots f_{N}=0, \quad \psi^{(x)}=0
$$

erhalten werden, $v+1$ unabhängige lineare Identitäten, woraus die Involutionseigenschaft des Systems (4) oder (2) sofort folgt.

35. Die eharakteristisehe Matrix des Involutionssystems (4) hat folgende Gestalt:

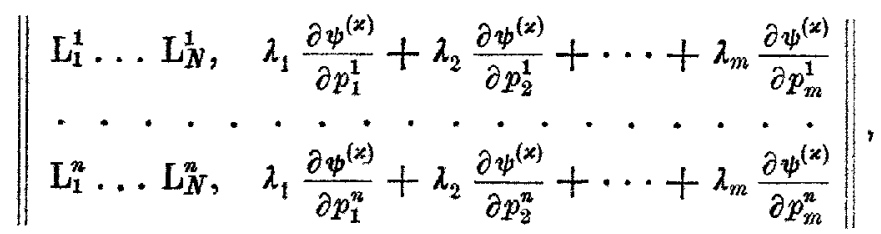

wobei in den Elementen der letzten Colonne die verschwindenden $A b$ leitungen $\frac{\partial \psi^{(x)}}{\partial p_{a}^{b}}$ nur der Symmetrie halber eingeführt wurden.

Aus (3) folgert man nun die für beliebige $\lambda$ bestehenden Identitäten:

$$
\begin{gathered}
\sum_{1}^{N}\left(\sigma_{i 1} \lambda_{1}+\cdots+\sigma_{i m} \lambda_{m}\right) L_{i}^{k} \\
-\left(\lambda_{1}+\Lambda_{2}^{(x)} \lambda_{2}+\cdots+\Lambda_{m}^{(x)} \lambda_{m}\right)\left(\lambda_{1} \frac{\partial \psi^{(x)}}{\partial p_{1}^{k}}+\cdots+\lambda_{m} \frac{\partial \psi^{(x)}}{\partial p_{m}^{k}}\right)=0 \\
(k=1,2 \ldots n) .
\end{gathered}
$$


In der Bezeichnungsweise der Nr. 15 schreibt sich demnach die zu (5) correspondirende Matrix so:

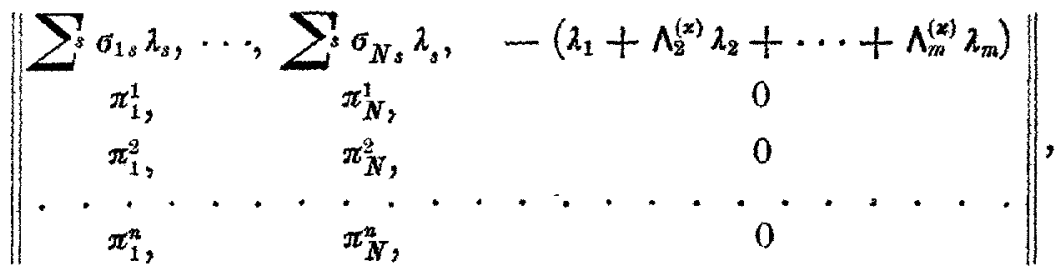

woraus sich exgibt, dass die charakteristische Form des Involutionssystems (4) erhalten wird, indem man aus $K$ den Factor $\Lambda_{x}$ weglässt.

36. Die Resultate der vor. Nr, lassen sich leicht verallgemeinern. Es sei $s<n-v$ und $x_{1} x_{2} \ldots x_{*}$ verschiedene Zahlen der Reihe $1,2 \ldots n-v$. Gestatten dann alie Systeme

$$
\left(\mathrm{H}_{x_{1}}^{\prime}\right),\left(\mathrm{H}_{x_{2}}^{\prime}\right) \ldots\left(\mathrm{H}_{x_{s}}^{\prime}\right)
$$

je eine integrable Combination $d \psi^{\left(x_{h}\right)}$ von der Beschaffenheit, dass die Gleichungen

$$
\psi^{\left(x_{3}\right)}=0, \quad \psi^{\left(x_{2}\right)}=0 \ldots \psi^{\left(x_{s}\right)}=0
$$

in deren linke Seiten die Variabeln $x, z, p_{2}^{*+1} \cdot p_{2}^{n}, p_{3}^{1} \cdots p_{m}^{n}$ eingehen, sich in der Form:

$$
-p_{2}^{p+1}+\varphi_{2}^{\nu+1}=0 \ldots-p_{2}^{v+s}+\varphi_{2}^{p+s}=0
$$

auflösen lassen, so stellen die Gleichungen:

$$
f_{1}=0, \ldots, f_{N}=0, \quad \psi^{\left(x_{1}\right)}=0, \ldots, \psi^{\left(x_{s}\right)}=0
$$

oder die damit äquivalenten Relationen (A') und (7) ein Involutionssystem dor, dessen charakteristische Form mit dem Ausdruck

identisch ist.

$$
\pm \frac{K}{\Lambda_{x_{1}} \Lambda_{x_{2}} \cdots \Lambda_{x_{s}}}
$$

37. Sind $u^{(x)}, v^{(x)}$ Integrale des Systems $\left(\mathrm{H}_{x}^{\prime}\right)$, so gilt dasselbe von jeder arbiträren Function $\varphi\left(u^{(x)}, v^{(x)}\right)$. Nehmen wir nun an, dass jedes dex s Gleichnngssysteme (6) je $m$ unabhängige Integrale $u_{i}^{\left(x_{h}\right)} u_{2}^{\left(x_{k}\right)} \ldots u_{m}^{\left(x_{k}\right)}$ besitze, und verstehen wir unter $\psi^{\left(x_{h}\right)}$ eine arbiträre Function dieser Ausdrücke, so bilden die Gleichungen (8) natürlich wieder ein Involutionssystem, und es ist klar, dass jede Integral- $M_{m}$ desselben anch das ursprüngliche System $J$ erfültt. Aber man kann anch umgekehrt die willkürlichen Functionen $\psi^{\left(x_{k}\right)}$ so bestimmen, dass eine beliebige Integral- $\boldsymbol{M}_{m}$ von $\boldsymbol{J}$ auch das System (8) befriedigt. Nach Nr. 10 ist nämlich füx jedes Involutionssystem eine Integral $-M_{m}$ durch Angabe einer sie enthaltenden Integral - $\boldsymbol{M}_{m-1}$ vollständig bestimmt. Wir denken uns nan eine beliebige Integral $-\mathcal{M}_{m-1}$ von $J$ ermittelt, was im Falle $v=0$ durch ansführbare Operationen, im Falle $v>0$ 
durch Integration eines Hülfssystems in $m-1$ Independenten geschiebt. Die Ausdrücke, die sich so für die Grössen $x, z^{1} \ldots z^{n}, p_{i}^{k}$ als Functionen der Variabeln $x_{2} \ldots x_{m}$ ergeben, denken wir uns in die Relationen $\psi^{\left(x_{k}\right)}=0$ substituirt. Man kann dann die arbiträren Functionen auf eine und wesentlich nur eine Art so wählen, dass diese Relationen identisch, $\mathrm{d}$. h. für beliebige Werthe der Variabeln $x_{2}, \ldots, x_{m}$ erfüllt sind, mit andern Worten: dass die erwäbnte Integral $-\boldsymbol{M}_{n-1}$ auch dem Involutionssystem (8) genügt. Die Herstellung der allgemeinsten Integral $-M_{m}$ von $J$ ist somit auf die Integration des $I n$ volutionssystems (8) zurückgeführt. Ist die Zahl $s$ insbesondere gleich $n-\nu$, so besteht das letztere System aus $2 n$ partiellen Differentialgleichungen in $n$ unbekannten Functionen; indem man wie in Nr. 11 in die aufgelöste Form

$$
-p_{a}^{b}+\varphi_{a}^{b}=0 \quad(a=1,2 ; b=1,2, \ldots, n)
$$

desselben vermöge der Mayer'schen Transformationsformeln $x_{1}=x_{1}^{0}+y_{1}, \quad x_{2}=x_{2}^{0}+y_{1} y_{2}, \quad x_{3}=x_{3}^{0}+y_{3} \ldots x_{m}=x_{m}^{0}+y_{m}$ neue unabhängige Veränderliche $y_{1} y_{2} \ldots y_{m}$ einfübrt, gelingt es, die Integration dieses Systems auf diejenige eines Systems ( $\left.\mathrm{A}^{\prime \prime}\right)$ von $n$ Gleichungen in $n$ abhängigen Veränderlichen $z^{1} \ldots z^{n}$ und $m-1$ Independenten $y_{1}, y_{3} \ldots y_{m}$ zurückzuführen.

Da man die Existenz etwaiger Integrale von $\left(\mathrm{H}_{*}^{\prime}\right)$ stets mit Hülfe von Differentiationen und Eliminationen feststellen, die-Integrale selbst durch Integration gewöhnlicher Differentialgleichungen ermitteln kann, so ergibt sich folgendes Theorem:

„Besitzt jedes der $n-\nu$. Systeme totaler Differentialgleichungen, durch welche bezw. die $n-\nu$ Systeme charaliteristischer Streifen unseres Normalsystems definirt werden, $m$ unabhängige Integrale, so kann die Integration des Normalsystems durch ausführbare Operationen und Integration gewöhnlicher Differentialgleichungssysteme auf Differentialprobleme in $m-1$ Independenten zurïckgeführt voerden, und es lässt sich stets durch Differentiationen und Eliminationen entscheiden, ob bei einem gegebenen Normalsystem dieser Ansatz zum Ziele führt oder nicht.*)"

München, im Januar 1897.

*) Weitere Resnitate dieser Theorie habe ich in einer Note: „Résumé einer Integrationstheorie höherer partieller Differentialprobleme“ Leipz. Ber. 1897 skizzirt. 